\title{
The orbital picture of the first dipole hyperpolarizability from many-body response theory
}

\author{
K.D. Nanda ${ }^{1, \text { a) }}$ and A.I. Krylov ${ }^{1, b)}$ \\ Department of Chemistry, University of Southern California, Los Angeles, California 90089-0482
}

(Dated: 2 March 2021)

We present an approach for obtaining a molecular orbital picture of the first dipole hyperpolarizability $(\beta)$ from correlated many-body electronic structure methods. Ab initio calculations of $\beta$ rely on quadratic response theory, which recasts the sum-over-all-states expression of $\beta$ into a closed-form expression by calculating a handful of first- and second-order response states; for resonantly enhanced $\beta$, damped response theory is used. These response states are then used to construct second-order response reduced one-particle density matrices (1PDMs), which, upon visualization in terms of natural orbitals (NOs), facilitate a rigorous and black-box mapping of the underlying electronic structure with $\beta$. We explain the interpretation of different components of the response 1PDMs and the corresponding NOs within both the undamped and damped response theory framework. We illustrate the utility of this new tool by deconstructing $\beta$ for cis-difluoroethene, para-nitroaniline, and hemibonded $\mathrm{OH}+\mathrm{H}_{2} \mathrm{O}$ complex, computed within the framework of coupled-cluster singles and doubles response theory, in terms of the underlying response 1PDMs and NOs for a range of frequencies.

\section{INTRODUCTION}

Nonlinear spectroscopic techniques ${ }^{1-6}$ provide a powerful set of tools to interrogate the structure of matter. Higherorder response properties are also exploited in applications such as optogenetics ${ }^{7}$, photodynamic cancer therapy ${ }^{8,9}$, photopharmacology $^{10}$, bioimaging ${ }^{11}$, optoelectronics ${ }^{12}$ and optical data storage $\mathrm{e}^{13,14}$. The spectroscopic signals are related to higher-order response properties of the system, which describe its nonlinear response to external fields.

The first dipole hyperpolarizability $(\beta)$ describes the thirdorder response of the energy $(E)$ of a system and the quadratic response of its dipole moment $(\mu)$ in the presence of electricfield perturbations $(\mathscr{E})$, according to the following Taylor series expansion:

$E(\mathscr{E})=E-\sum_{a} \mu^{a} \mathscr{E}_{a}-\frac{1}{2} \sum_{a b} \alpha^{a b} \mathscr{E}_{a} \mathscr{E}_{b}-\frac{1}{6} \sum \beta^{a b c} \mathscr{E}_{a} \mathscr{E}_{b} \mathscr{E}_{c}-\cdots$

with $E=E(0)$ is the energy of the system in the absence of these perturbations, $\alpha$ is the polarizability, and $a, b$, and $c$ are the Cartesian components of the perturbation. For exact states within the many-body perturbation theory, $\beta$ for state $k$ is given by the following sum-over-states (SOS) expression ${ }^{15,16}$ :

$$
\begin{gathered}
\beta_{k}^{a b c}\left(\omega_{a} ; \omega_{b}, \omega_{c}\right)=\frac{1}{2} \mathcal{C}_{ \pm \omega} \mathcal{P}_{+}^{a b c} \sum_{m \neq k, n \neq k} \\
\frac{\left\langle\Psi_{k}\left|\hat{\mu}^{a}\right| \Psi_{m}\right\rangle\left\langle\Psi_{m}\left|\overline{\hat{\mu}}_{k}^{b}\right| \Psi_{n}\right\rangle\left\langle\Psi_{n}\left|\hat{\mu}^{c}\right| \Psi_{k}\right\rangle}{\left(E_{m}-E_{k}+\omega_{a}\right)\left(E_{n}-E_{k}-\omega_{c}\right)}
\end{gathered}
$$

where $\omega_{b}$ and $\omega_{c}$ are the energies of the absorbed photons (polarized, respectively, along $\hat{b}$ and $\hat{c}$ directions) and $\omega_{a}=-\omega_{b}-\omega_{c}$ is the emitted sum-frequency photon's energy (polarized along $\hat{a}$ direction); $\hat{\mu}$ is the dipole operator;

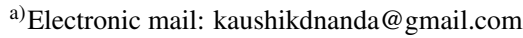

b)Electronic mail: krylov@usc.edu
}

$\overline{\hat{\mu}}_{k}=\hat{\mu}-\left\langle\Psi_{k}|\hat{\mu}| \Psi_{k}\right\rangle$ is the dipole fluctuation operator; $\Psi_{m}$ is the $m^{\text {th }}$ eigenstate with energy $E_{m}$ of the zero-order Hamiltonian $H$ without external perturbations; the operator $\frac{1}{2} \mathcal{C}_{ \pm}$involves complex conjugation and a simultaneous sign reversal of all frequencies; and $\mathcal{P}_{a b c}^{+}$is the symmetrizer with respect to indices $a, b$ and $c$. $\beta$ is related to such physical phenomena as the electro-optical Pockels effect ${ }^{17}\left(\beta^{a b c}(-\omega ; \omega, 0)\right)$, optical rectification $^{18}\left(\beta^{a b c}(0 ; \omega,-\omega)\right)$, and second harmonic generation $^{19}\left(\beta^{a b c}(-2 \omega ; \omega, \omega)\right)$.

$\beta^{a b c}\left(-\omega_{1}-\omega_{2} ; \omega_{1}, \omega_{2}\right)$ is also the underlying measurable quantity in electronic sum-frequency generation (SFG) spectroscopy ${ }^{3,4,17}$. SFG is a surface-sensitive nonlinear spectroscopy exploiting the macroscopic asymmetry introduced by the interface. The SFG process can be comprehended as a sum of a two-photon absorption from the initial state to the two-photon virtual state (upper dashed line in Fig. 1(a)) via the one-photon virtual state (lower dashed line in Fig. 1(a)) and an emission of the sum-frequency photon from the twophoton virtual state to the initial state. The SFG signal can be enhanced by tuning the energies of the absorbed photons or their sum or both to be resonant with an excitation energy or energies of the system (one-photon or two-photon or double resonant SFG, as shown in Figs. 1(b), 1(c), and 1(d)). The enhancement arises due to small $E_{m}-E_{k}+\omega$ and/or $E_{n}-E_{k}-\omega$ in the denominator(s) of SOS term(s) in Eq. (2).

Driven by the desire to identify molecules with large $\beta$ and to tailor them for various applications in nonlinear optics (e.g., optoelectronics and photonics), theoretical and computational attention has mainly focused on developing accurate methods. In addition, many studies have also focused on understanding the design principles that facilitate the development of tailored molecules. For example, recently, the impact of open-shell

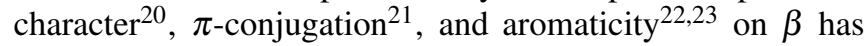
been studied. In the context of SFG spectroscopy, understanding the impact of solvent ${ }^{24,25}$ on $\beta$ is crucial for characterizing spectral features.

Fundamental understanding of nonlinear response and practical understanding of structural factors determining $\beta$ hinge on our ability to characterize the underlying one- and 


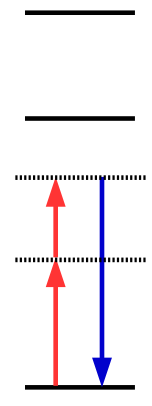

(a)

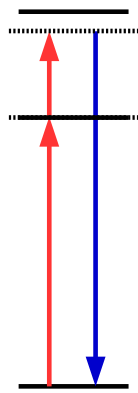

(b)

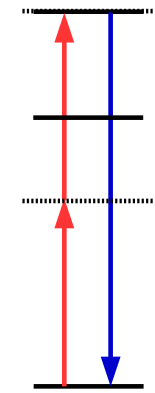

(c)

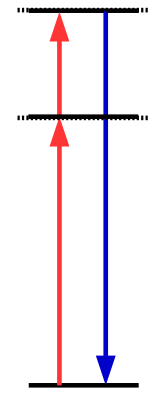

(d)
FIG. 1. Schematic representation of different SFG processes in terms of many-body states. (a) Non-resonant SFG. (b) One-photon resonant SFG. (c) Two-photon resonant SFG. (d) Doubly (one-photon and two-photon) resonant SFG. The solid lines represent zero-order electronic states (e.g., $\Psi_{k}$ in Eq. (2)). The dashed lines represent virtual states. In few-state models of resonant SFG, the virtual states are commonly approximated by zero-order states. In this paper, we show how these virtual states can be characterized in terms of transitions between zero-order states and second-order response states and between first-order response states, without making few-state approximations.

two-photon virtual states shown schematically in Fig. 1. A formidable roadblock in understanding molecular nonlinear property such as $\beta$ in terms of molecular orbitals is its formal SOS expressions in Eq. (2). Formally, all electronic states of the system contribute to $\beta$. Several studies that aimed to understand how the electronic structure relates to $\beta$ employed few-state models, wherein the SOS is truncated on the basis of near-resonance considerations. The accuracy of such models hinges on the choice of electronic states included in the SOS. In few-state models of resonant SFG, the virtual one- and twophoton states are approximated by zero-order states. Even though such models can provide a qualitative description of $\beta$, a priori evaluation of the error introduced from truncation is difficult. A similar challenge of deriving a molecular orbital picture from truncated SOS expressions applies to other nonlinear properties such as two-photon absorption (2PA) and resonant inelastic X-ray scattering (RIXS) cross sections and polarizabilities.

In this contribution, we extend our framework for computing orbitals associated with nonlinear processes such as $2 \mathrm{PA}^{26}$ and $\mathrm{RIXS}^{27}$ and introduce a novel approach of deriving the mechanistic details of $\beta$ by means of computing the response reduced one-particle density matrices (1PDMs) and their analyses in terms of natural orbitals (NOs). By ascribing orbitals to the transitions in Fig. 1, our approach enables characterization of the one- and two-photon virtual states in the SFG process, providing a black-box procedure of mapping $\beta$ onto molecular orbitals ${ }^{28}$. Our scheme does not invoke arbitrary truncation of the SOS expressions and is orbital invariant, in contrast to few-state models. The response 1PDMs are constructed by computing zero-, first-, and second-order frequency-dependent response states including the complex-valued response states from damped response theory (often used for computing response properties in the resonant regimes). The approach builds on our prior work on characterizing 2PA and RIXS cross sections by using response transition 1PDMs. The novelty lies in its extension to a thirdorder (or quadratic response) property such as $\beta$. Using exemplary systems such as cis-difluoroethene, para-nitroaniline, and hemibonded $\mathrm{OH}+\mathrm{H}_{2} \mathrm{O}$ complex, we demonstrate how the NOs computed from response 1PDMs facilitate the construction of orbital channels depicting the underlying change in the electronic distribution during the molecular process (e.g., $\mathrm{SFG}$ ) of $\beta$. For the complex-valued response 1PDMs from damped response theory, we also explain the contributions of real and imaginary parts and their respective NOs. We also show how these response 1PDMs provide characteristic quantities such as the degree of delocalization, charge separation, and charge transfer in the context of the SFG process.

\section{THEORY}

\section{A. Reduced density matrices and natural orbitals}

As stated above, our goal is to extend rigorous molecular orbital analysis, which is commonly used in quantum chemistry $^{28}$, to $\beta^{a b c}$. We begin by reviewing the essential elements of the theory. The key object, which allows mapping between many-body states $(\Psi)$ and orbitals is reduced one-particle density matrix $\gamma$

$$
\gamma_{p q}=\left\langle\Psi\left|p^{\dagger} q\right| \Psi\right\rangle
$$

where $p^{\dagger}$ and $q$ are electron-creation and electron-annihilation ) operators corresponding to orbitals $\phi_{p}$ and $\phi_{q}$, respectively. Although $\gamma$ is a much more compact object that the original many-body wave function, it contains all essential information needed to compute any one-electron property because an expectation value of a one-electron operator $\hat{\mathcal{O}}$ is simply:

$$
\langle\hat{\mathcal{O}}\rangle=\sum_{p q} \hat{\mathcal{O}}_{p q} \gamma_{p q}
$$

For example, the dipole moment is given by

$$
\langle\Psi|\hat{\mu}| \Psi\rangle=\sum_{p q} \hat{\mu}_{p q} \gamma_{p q}
$$

In a similar fashion, reduced one-particle density matrices can be defined for a pair of states $\Psi_{k}$ and $\Psi_{f}$ and then used to compute one-electron transition properties. The transition 1PDM $\gamma^{k \rightarrow f}$ provides a mapping between the two states in terms of one-electron excitations ${ }^{28,29}$ :

$$
\Psi_{f}=\sum_{p q} \gamma_{p q}^{k \rightarrow f} p^{\dagger} q\left|\Psi_{k}\right\rangle+\text { higher excitations. }
$$

Using $\gamma^{k \rightarrow f}$, one can define exciton's wave function $\Psi_{\text {exc }}$ as $^{30}$

$$
\Psi_{\mathrm{exc}}\left(r_{h}, r_{e}\right)=\sum_{p q} \gamma_{p q}^{k \rightarrow f} \phi_{p}\left(r_{e}\right) \phi_{q}\left(r_{h}\right),
$$

where $r_{h}$ and $r_{e}$ are the hole and electron (particle) coordinates and $\phi$ are molecular orbitals. This object is a compact representation of the electronic transition and its expectation values 
are exactly equal to the matrix elements between the original many-body states from which it is derived.

State and transition 1PDMs can be further compressed by representing them in the basis of their eigenstates. For example, by diagonalizing the state 1PDM one obtains natural orbitals (NOs). An analogous transformation for the transition 1PDM is given by the singular value decomposition (SVD) procedure, which defines natural transition orbitals (NTOs). NO and NTOs provide the most compact description of manybody states and the transitions between them. Because they are related to molecular properties, NOs and NTOs represent physically meaningful orbital description of electronic structure $^{29-36}$.

NTOs are obtained from unitary transformation of orbitals $\phi$ s by means of SVD

$$
\gamma=\mathbf{V} \Sigma \mathbf{U}^{\mathbf{T}}
$$

Here, $\mathbf{U}$ and $\mathbf{V}$ contain the hole and particle NOs $\left(\psi_{S}^{h} \mathbf{s}\right.$ and $\left.\psi_{S}^{e} \mathrm{~s}\right)$ and $\Sigma$ is the diagonal matrix of singular values $\left(\sigma_{S} \mathrm{~s}\right)$ such that

$$
\begin{aligned}
& \psi_{S}^{h}(r)=\sum_{q} U_{q S} \phi_{q}(r), \\
& \psi_{S}^{e}(r)=\sum_{p} V_{p S} \phi_{p}(r),
\end{aligned}
$$

Using NTOs, one can express molecular properties as matrix elements between hole and pair orbitals. Using dipole moment as an example:

$$
\left\langle\Psi_{f}|\hat{\mu}| \Psi_{k}\right\rangle=\sum_{S} \sigma_{S}\left\langle\psi_{S}^{p}|\mu| \psi_{S}^{h}\right\rangle .
$$

In this representation, one can, for example, visualize and interpret selection rules between many-body states in terms of transitions between orbitals. Hence, one of the benefits of finding similar representation for $\beta$ (i.e., by expressing it in terms of a response 1PDM and $\hat{\mu}$ ) is that it would allow us to deconstruct its underlying symmetries and to understand the selection rules in the same fashion as was done for one- and two-photon processes ${ }^{26,27,30,34,35,37,38}$; the symmetries originate in the polarization of the three photons $(a, b$, and $c)$.

\section{B. Expressing $\beta$ in terms of response states}

$\beta^{a b c}$ in Eq. (2) has poles when the energies of the photons match the excitation energies of electronic states relative to state $k$. For such resonant cases, an alternate SOS formulation of $\beta$ based on the Ehrenfest theorem ${ }^{39,40}$, wherein the excitation energies $E_{m}-E_{k}$ are augmented by imaginary inverse lifetimes $\varepsilon_{m}$, is given according to

$$
\begin{aligned}
& \beta_{k}^{a b c}\left(\omega_{a} ; \omega_{b}, \omega_{c}\right)=\frac{1}{2} \mathcal{C}_{ \pm \omega} \mathcal{P}_{+}^{b c} \sum_{m \neq k, n \neq k} \\
& \left(\frac{\left\langle\Psi_{k}\left|\hat{\mu}^{a}\right| \Psi_{m}\right\rangle\left\langle\Psi_{m}\left|\overline{\hat{\mu}}_{k}^{b}\right| \Psi_{n}\right\rangle\left\langle\Psi_{n}\left|\hat{\mu}^{c}\right| \Psi_{k}\right\rangle}{\left(E_{m}-E_{k}+\omega_{a}-i \varepsilon_{m}\right)\left(E_{n}-E_{k}-\omega_{c}-i \varepsilon_{n}\right)}\right. \\
& +\frac{\left\langle\Psi_{k}\left|\hat{\mu}^{b}\right| \Psi_{m}\right\rangle\left\langle\Psi_{m}\left|\overline{\hat{\mu}}_{k}^{a}\right| \Psi_{n}\right\rangle\left\langle\Psi_{n}\left|\hat{\mu}^{c}\right| \Psi_{k}\right\rangle}{\left(E_{m}-E_{k}+\omega_{b}+i \varepsilon_{m}\right)\left(E_{n}-E_{k}-\omega_{c}-i \varepsilon_{n}\right)} \\
& \left.+\frac{\left\langle\Psi_{k}\left|\hat{\mu}^{c}\right| \Psi_{m}\right\rangle\left\langle\Psi_{m}\left|\overline{\hat{\mu}}_{k}^{b}\right| \Psi_{n}\right\rangle\left\langle\Psi_{n}\left|\hat{\mu}^{a}\right| \Psi_{k}\right\rangle}{\left(E_{m}-E_{k}+\omega_{c}+i \varepsilon_{m}\right)\left(E_{n}-E_{k}-\omega_{a}+i \varepsilon_{n}\right)}\right),
\end{aligned}
$$

where $\mathcal{P}_{b c}^{+}$is the symmetrizer with respect to indices $b$ and $c$. Eq. (12) reduces to Eq. (2) if $\varepsilon^{m}=0 \forall$ states $m$; so, we only discuss the theory based on Eq. (12) below. For a Hermitian theory (either exact or approximate), $\beta^{a b c}$ is symmetric with respect to complex conjugation and signs of photon frequencies; this, however, is not true for non-Hermitian methods (e.g., equation-of-motion coupled-cluster theory ${ }^{41,42}$ ) for which the left and the right transition moments are not equivalent.

It is impractical to compute $\beta$ directly from Eq. (12) by computing all electronic states and their excitation energies and dipole and transition moments relative to the state $k$ beyond small systems and basis sets. Many-body damped response theory provides a more compact representation of the SOS expression in terms of response wave functions, which is the approach taken in our implementation of $\beta$ in the Q-Chem package $^{43,44}$. Within damped response theory ${ }^{45-47}$, a phenomenological damping factor $\varepsilon$ replaces the inverse lifetimes $\varepsilon_{m} \forall$ states $m$. Fig. 2 explains the effect of this phenomenological damping on the individual SOS terms of Eq. (12). The effect of the $i \varepsilon$ varies for individual SOS terms depending on whether the two photons $\left(\omega^{\prime}, \omega^{\prime \prime} \in\left\{\omega_{a}, \omega_{b}, \omega_{c}\right\}\right)$ are nearly resonant or off resonant with the corresponding excitation energies $E_{m}-E_{k}$ and $E_{n}-E_{k}$ in the denominators or whether one of the photons is nearly resonant and the other is off resonant. The ranges for the two photons for being nearly resonant are explained in Fig. 2. When both photons are far from being resonant with the two excitation energies such that $\varepsilon<\Omega_{n k}-$ $\omega^{\prime \prime}=\chi \varepsilon$ and $\Omega_{m k}-\omega^{\prime} \notin\left\{\frac{\varepsilon\left(s^{\prime} s^{\prime \prime}-\chi s^{\prime}\right)}{\left(s^{\prime \prime}+\chi\right)}, \frac{\varepsilon\left(s^{\prime} s^{\prime \prime}+\chi s^{\prime}\right)}{\left(\chi-s^{\prime \prime}\right)}\right\}$ (Fig. 2(f)), the real part of the corresponding complex SOS term dominates and the imaginary component is negligible. This is also true when both photons are nearly resonant with the corresponding excitation energies such that $0<\Omega_{n k}-\omega^{\prime \prime}=\chi \varepsilon<\varepsilon$ and $\Omega_{m k}-\omega^{\prime} \in\left\{\frac{\varepsilon\left(s^{\prime} s^{\prime \prime}+\chi s^{\prime}\right)}{\left(\chi-s^{\prime \prime}\right)}, \frac{\varepsilon\left(s^{\prime} s^{\prime \prime}-\chi s^{\prime}\right)}{\left(s^{\prime \prime}+\chi\right)}\right\}$ (Fig. 2(e)). The latter situation arises when the underlying molecular process of $\beta$ involves a double resonance enhancement. However, when only one of the two photons is nearly resonant with the corresponding excitation energy such that $0<\Omega_{n k}-\omega^{\prime \prime}=\chi \varepsilon<\varepsilon$ and $\Omega_{m k}-\omega^{\prime} \notin\left\{\frac{\varepsilon\left(s^{\prime} s^{\prime \prime}+\chi s^{\prime}\right)}{\left(\chi-s^{\prime \prime}\right)}, \frac{\varepsilon\left(s^{\prime} s^{\prime \prime}-\chi s^{\prime}\right)}{\left(s^{\prime \prime}+\chi\right)}\right\}$, the contribution to the imaginary component of the SOS term dominates.

Following the introduction of the phenomenological damping, Eq. (12) is recast into a sum of expectation values between different complex-valued first-order response wave 

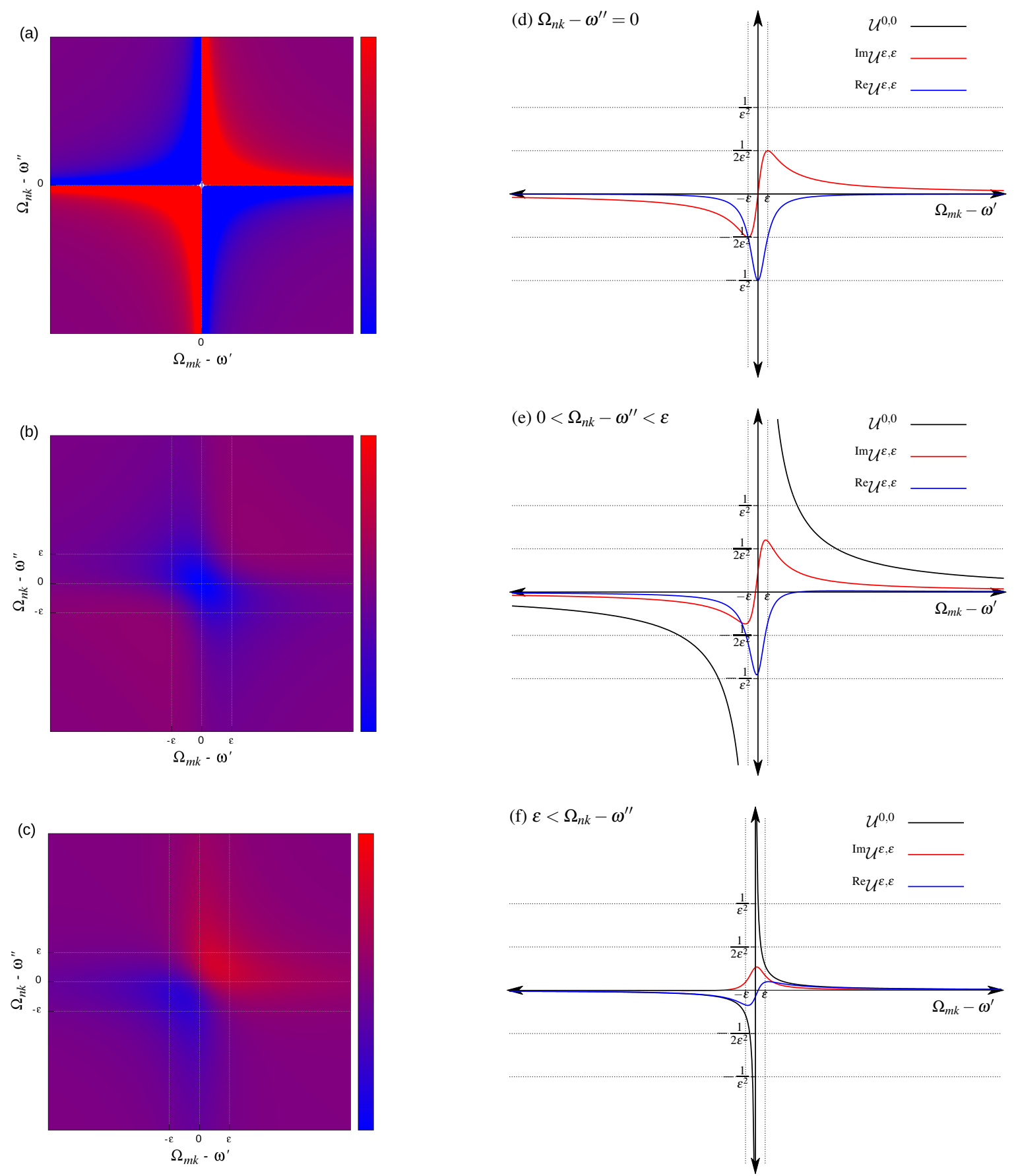

FIG. 2. Effect of the imaginary phenomenological damping $i \varepsilon(\varepsilon>0)$ on $\beta$. The individual SOS terms are proportional to $\frac{1}{\left(\Omega_{m k}-\omega^{\prime}-i \varepsilon^{\prime}\right)\left(\Omega_{n k}-\omega^{\prime \prime}-i \varepsilon^{\prime \prime}\right)}=\mathcal{U}^{\varepsilon^{\prime}, \varepsilon^{\prime \prime}}\left(\Omega_{m k}-\omega^{\prime}, \Omega_{n k}-\omega^{\prime \prime}\right)$, where $\Omega_{m k}=E_{m}-E_{k}, s^{\prime} \varepsilon^{\prime}=s^{\prime \prime} \varepsilon^{\prime \prime}=\varepsilon$, sign $\left(\varepsilon^{\prime}\right)=s^{\prime}$, and sign $\left(\varepsilon^{\prime \prime}\right)=s^{\prime \prime}$. Since the different signs of the two variables, $\varepsilon^{\prime}$, and $\varepsilon^{\prime \prime}$ give similar information regarding the corresponding $\operatorname{Re} \mathcal{U}^{\varepsilon^{\prime}, \varepsilon^{\prime \prime}}$ and $\operatorname{Im} \mathcal{U}^{\varepsilon^{\prime}, \varepsilon^{\prime \prime}}$, here, only the two-dimensional maps of $\mathcal{U}^{0,0},{ }^{\operatorname{Re}} \mathcal{U}^{\varepsilon, \varepsilon}$, and ${ }^{\operatorname{Im}} \mathcal{U}^{\varepsilon, \varepsilon}$ are shown in (a), (b), and (c), respectively. $\mathcal{U}^{0,0}$ has poles when one or both of the photon energies, $\omega^{\prime}$ and $\omega^{\prime \prime}$, are resonant with energy differences, $\Omega_{m k}$ and $\Omega_{n k}$, respectively. Nonzero $i \varepsilon$ brings these poles into the complex plane, preventing the SOS terms from being indeterminate. Far from the poles, $\mathcal{U}^{0,0}$ and ${ }^{R e} \mathcal{U}^{\varepsilon, \varepsilon}$ give similar results. In (d), (e), and (f), slices of ${ }^{R e} \mathcal{U}^{\varepsilon, \varepsilon}$ (blue) and $\operatorname{Im}_{\mathcal{U}^{\varepsilon, \varepsilon}}$ (red) are shown for different values of the variable $\Omega_{n k}-\omega^{\prime \prime}$. (d) $\Omega_{n k}-\omega^{\prime \prime}=0: \mathcal{U}^{0,0}$ is indeterminate; on the other hand, ${ }^{\operatorname{Re}} \mathcal{U}^{\varepsilon, \varepsilon}(0,0)=-\frac{1}{\varepsilon^{2}}$ and ${ }^{\operatorname{Im}} \mathcal{U}^{\varepsilon, \varepsilon}(0,0)=0$. For $\left|\Omega_{m k}-\omega^{\prime}\right|<\varepsilon$, the magnitude of ${ }^{\operatorname{Im}} \mathcal{U}^{\varepsilon, \varepsilon}$ is smaller than ${ }^{R e} \mathcal{U}^{\varepsilon, \varepsilon}$, indicating that $i \varepsilon$ brings the contribution of the SOS term predominantly into the real component when both photon energies are (nearly) resonant with $\Omega_{m k}$ and $\Omega_{n k}$. For $\left|\Omega_{m k}-\omega^{\prime}\right|>\varepsilon$, the contribution of the SOS term predominantly goes into ${ }^{\operatorname{Im}} \mathcal{U}^{\varepsilon, \varepsilon}$. (e) $0<\Omega_{n k}-\omega^{\prime \prime}=\chi \varepsilon<\varepsilon$ : For $\Omega_{m k}-\omega^{\prime} \in\left\{\frac{\varepsilon\left(s^{\prime} s^{\prime \prime}+\chi s^{\prime}\right)}{\left(\chi-s^{\prime \prime}\right)}, \frac{\varepsilon\left(s^{\prime} s^{\prime \prime}-\chi s^{\prime}\right)}{\left(s^{\prime \prime}+\chi\right)}\right\}$, the damping brings the contribution of the SOS term predominantly into Re $\mathcal{U}^{\varepsilon, \varepsilon}$; else, the contribution of the SOS term predominantly goes into $\operatorname{Im} \mathcal{U}^{\varepsilon, \varepsilon}$. (f) $\varepsilon<\Omega_{n k}-\omega^{\prime \prime}=\chi \varepsilon$ : For $\Omega_{m k}-\omega^{\prime} \in\left\{\frac{\varepsilon\left(s^{\prime} s^{\prime \prime}-\chi s^{\prime}\right)}{\left(s^{\prime \prime}+\chi\right)}, \frac{\varepsilon\left(s^{\prime} s^{\prime \prime}+\chi s^{\prime}\right)}{\left(\chi-s^{\prime \prime}\right)}\right\}$, the damping brings the contribution of the SOS term predominantly into $\operatorname{Im} \mathcal{U}^{\varepsilon, \varepsilon}$; else, the contribution of the SOS term predominantly goes into ${ }^{\operatorname{Re}} \mathcal{U}^{\varepsilon, \varepsilon}$. 
functions $\tilde{X}_{\omega_{a}}^{a}$ and $X_{\omega_{a}}^{a}$ as follows ${ }^{26,27,48,49}$ :

$$
\begin{aligned}
\beta_{k}^{a b c} & \left(\omega_{a} ; \omega_{b}, \omega_{c} ; \varepsilon\right) \\
=\frac{1}{2} & \mathcal{C}_{ \pm \omega} \mathcal{P}_{+}^{b c}\left(\left\langle\tilde{X}_{k ;-\varepsilon, \omega_{a}}^{a}\left|\overline{\hat{\mu}}_{k}^{b}\right| X_{k ; \varepsilon, \omega_{c}}^{c}\right\rangle\right. \\
& +\left\langle\tilde{X}_{k ; \varepsilon, \omega_{b}}^{b}\left|\overline{\hat{\mu}}_{k}^{a}\right| X_{k ; \varepsilon, \omega_{c}}^{c}\right\rangle \\
& \left.+\left\langle\tilde{X}_{k ; \varepsilon, \omega_{c}}^{c}\left|\overline{\hat{\mu}}_{k}^{b}\right| X_{k ;-\varepsilon, \omega_{a}}^{a}\right\rangle\right)
\end{aligned}
$$

These response wave functions are computed by solving the following response equations:

$$
\begin{aligned}
& \sum_{V}\left\langle\tilde{X}_{k ; \varepsilon, \omega_{a}}^{a} \mid \Phi_{v}\right\rangle\left\langle\Phi_{v}\left|\left(H_{k}^{e f f}-E_{k}+\omega_{a}+i \varepsilon\right)\right| \Phi_{\rho}\right\rangle \\
& \quad=\left\langle\tilde{D}_{k}^{a} \mid \Phi_{\rho}\right\rangle-\left\langle\tilde{D}_{k}^{a} \mid \Psi_{k}\right\rangle\left\langle\Psi_{k} \mid \Phi_{\rho}\right\rangle
\end{aligned}
$$

and

$$
\begin{aligned}
& \sum_{\rho}\left\langle\Phi_{v}\left|\left(H_{k}^{e f f}-E_{k}-\omega_{a}-i \varepsilon\right)\right| \Phi_{\rho}\right\rangle\left\langle\Phi_{\rho} \mid X_{k ; \varepsilon, \omega_{a}}^{a}\right\rangle \\
& \quad=\left\langle\Phi_{v} \mid D_{k}^{a}\right\rangle-\left\langle\Phi_{v} \mid \Psi_{k}\right\rangle\left\langle\Psi_{k} \mid D_{k}^{a}\right\rangle .
\end{aligned}
$$

In Eqs. (14) and (15), $H_{k}^{\text {eff }}$ is the Hamiltonian $H$ with the state $k$ projected out according to

$$
\begin{aligned}
& \left\langle\Phi_{V}\left|H_{k}^{e f f}\right| \Phi_{\rho}\right\rangle= \\
& \quad\left\langle\Phi_{V}\left|\left(1-\left|\Psi_{k}\right\rangle\left\langle\Psi_{k}\right|\right) H\left(1-\left|\Psi_{k}\right\rangle\left\langle\Psi_{k}\right|\right)\right| \Phi_{\rho}\right\rangle,
\end{aligned}
$$

where both $v$ and $\rho$ span the configuration manifold. The amplitudes of intermediates $\tilde{D}^{a}$ and $D^{a}$ are

$$
\left\langle\tilde{D}_{k}^{a} \mid \Phi_{\rho}\right\rangle=\left\langle\Psi_{k}\left|\hat{\mu}^{a}\right| \Phi_{\rho}\right\rangle
$$

and

$$
\left\langle\Phi_{v} \mid D_{k}^{a}\right\rangle=\left\langle\Phi_{v}\left|\hat{\mu}^{a}\right| \Psi_{k}\right\rangle
$$

In the discussion below, we drop the state index $k$ for brevity.

\section{From resonse states to response density matrices and NTOs}

Eq. (13) gives $\beta^{a b c}$ as sum of expectation values between different pairs of first-order response wave functions. Despite its closed form, $\beta^{a b c}$ in Eq. (13) cannot be expressed using a single response 1PDM $(\gamma)$ contracted with an operator, as per Eq. (3). In order to express $\beta^{a b c}$ using a single response 1PDM, we compute second-order response states $\tilde{X}_{\omega_{c},-\omega_{a}}^{c b}$ and $X_{-\omega_{a}, \omega_{c}}^{b c}$ using Eqs. (14) and (15) such that the second-order response intermediates $\tilde{D}_{\omega_{c}}^{b}$ and $D_{\omega_{c}}^{b}$ are used instead of $\tilde{D}^{a}$ and $D^{a}$, respectively. The amplitudes of $\tilde{D}_{\omega_{c}}^{b}$ and $D_{\omega_{c}}^{b}$ are given in terms of first-order response wave functions as follows:

$$
\left\langle\tilde{D}_{\varepsilon, \omega_{c}}^{b} \mid \Phi_{\rho}\right\rangle=\left\langle\tilde{X}_{\varepsilon, \omega_{c}}^{c}\left|\overline{\hat{\mu}}^{b}\right| \Phi_{\rho}\right\rangle
$$

and

$$
\left\langle\Phi_{v} \mid D_{\varepsilon, \omega_{c}}^{b}\right\rangle=\left\langle\Phi_{v}\left|\overline{\hat{\mu}}^{b}\right| X_{\varepsilon, \omega_{c}}^{c}\right\rangle .
$$

$\beta^{a b c}$ can now be expressed as

$$
\beta^{a b c}\left(\omega_{a} ; \omega_{b}, \omega_{c} ; \varepsilon\right)=\sum_{p q} \hat{\mu}_{p q}^{a}\left[\gamma_{\varepsilon, \omega_{b}, \omega_{c}}^{b c}\right]_{p q},
$$

where the $b c$-component response $1 \mathrm{PDM} \gamma_{\varepsilon, \omega_{b}, \omega_{c}}$ is given by

$$
\begin{aligned}
& {\left[\gamma_{\varepsilon, \omega_{b}, \omega_{c}}^{b c}\right]_{p q}=\frac{1}{2} \mathcal{C}_{ \pm \omega}\left(\left\langle\Psi\left|p^{\dagger} q\right| X_{\varepsilon,-\omega_{a} ; \varepsilon, \omega_{c}}^{b c}\right\rangle\right.} \\
& \quad+\left\langle\tilde{X}_{\varepsilon, \omega_{b}}^{b}\left|\left(p^{\dagger} q-\gamma_{p q}\right)\right| X_{\varepsilon, \omega_{c}}^{c}\right\rangle \\
& \quad+\left\langle\tilde{X}_{\left.\varepsilon, \omega_{c} ; \varepsilon,-\omega_{a}\left|p^{\dagger} q\right| \Psi\right\rangle}^{c b}\right. \\
& \quad+\left\langle\Psi\left|p^{\dagger} q\right| X_{\varepsilon,-\omega_{a} ; \varepsilon, \omega_{b}}^{c b}\right\rangle \\
& \quad+\left\langle\tilde{X}_{\varepsilon, \omega_{c}}^{c}\left|\left(p^{\dagger} q-\gamma_{p q}\right)\right| X_{\varepsilon, \omega_{b}}^{b}\right\rangle \\
& \left.\quad+\left\langle\tilde{X}_{\varepsilon, \omega_{b} ; \varepsilon,-\omega_{a}}^{b c}\left|p^{\dagger} q\right| \Psi\right\rangle\right) \\
& =\left[\gamma_{\varepsilon, \omega_{b}, \omega_{c}}^{b c,+}\right]_{p q}+\left[\gamma_{\varepsilon, \omega_{b}, \omega_{c}}^{b c,-}\right]_{p q} .
\end{aligned}
$$

The above construction of the second-order response 1PDM is not unique. $\beta^{a b c}$ can also be expressed using an alternative construction for the $a c$-component response 1PDM according to

$$
\beta^{a b c}\left(\omega_{a} ; \omega_{b}, \omega_{c} ; \varepsilon\right)=\sum_{p q} \hat{\mu}_{p q}^{b}\left[\tilde{\gamma}_{\varepsilon, \omega_{b}, \omega_{c}}^{a c}\right]_{p q}
$$

where

$$
\begin{aligned}
& {\left[\tilde{\gamma}_{\varepsilon, \omega_{b}, \omega_{c}}^{a c}\right]_{p q}=\frac{1}{2} \mathcal{C}_{ \pm \omega}\left(\left\langle\tilde{X}_{-\varepsilon, \omega_{a}}^{a}\left|\left(p^{\dagger} q-\gamma_{p q}\right)\right| X_{\varepsilon, \omega_{c}}^{c}\right\rangle\right.} \\
& \quad+\left\langle\Psi\left|p^{\dagger} q\right| X_{-\varepsilon,-\omega_{b} ; \varepsilon, \omega_{c}}^{a c}\right\rangle \\
& \quad+\left\langle\tilde{X}_{\varepsilon, \omega_{c}}^{c}\left|\left(p^{\dagger} q-\gamma_{p q}\right)\right| X_{-\varepsilon, \omega_{a}}^{a}\right\rangle \\
& \quad+\left\langle\tilde{X}_{-\varepsilon, \omega_{a} ;-\varepsilon,-\omega_{b}}^{a c}\left|p^{\dagger} q\right| \Psi\right\rangle \\
& \quad+\left\langle\tilde{X}_{\varepsilon, \omega_{c} ;-\varepsilon,-\omega_{b}}^{c a}\left|p^{\dagger} q\right| \Psi\right\rangle \\
& \left.\quad+\left\langle\Psi\left|p^{\dagger} q\right| X_{-\varepsilon,-\omega_{b} ;-\varepsilon, \omega_{a}}^{c a}\right\rangle\right) \\
& =\left[\tilde{\gamma}_{\varepsilon, \omega_{b}, \omega_{c}}^{a c,+}\right]_{p q}+\left[\tilde{\gamma}_{\varepsilon, \omega_{b}, \omega_{c}}^{a c,-}\right]_{p q}
\end{aligned}
$$

The response 1PDMs $\gamma_{\varepsilon, \omega_{1}, \omega_{2}}^{c c,+}$ and $\gamma_{\varepsilon, \omega_{1}, \omega_{2}}^{p c,-}\left(\tilde{\gamma}_{\varepsilon, \omega_{1}, \omega_{2}}^{a c,+}\right.$ and $\left.\tilde{\gamma}_{\varepsilon, \omega_{1}, \omega_{2}}^{a c,-}\right)$, obtained by the action of the operator $\frac{1}{2} \mathcal{C}_{ \pm \omega}$, are not symmetric (except for the static case), in contrast to the full second-order response 1PDM $\gamma_{\varepsilon, \omega_{1}, \omega_{2}}^{b c}\left(\tilde{\gamma}_{\varepsilon, \omega_{1}, \omega_{2}}^{a c}\right)$.

This non-uniqueness in the construction of the secondorder response 1PDM corresponding to $\beta$ is a consequence of the coherent nature of the underlying three-photon process. There are multiple ways in which one can describe the perturbed electronic density resulting from the interaction of the molecule with any two of the three photons, followed by the third photon. For example, for the SFG $\beta$, Eq. (21) can be construed as an electronic density in Eq. (22), resulting from the interaction of the two absorbed photons, interacting with the emitted photon. Similarly, the underlying process of $\beta$ in 
Eq. (23) can be construed as an electronic density in (24), resulting from the interaction of the molecule with one of the absorbed photons and the emitted photon, interacting with the other absorbed photon.

$\gamma_{\varepsilon, \omega_{1}, \omega_{2}}^{b c}$ and $\tilde{\gamma}_{\varepsilon, \omega_{1}, \omega_{2}}^{a c}$ are state-specific complex-valued quantities; each is expressed as a sum of constituent $\omega \mathrm{DMs}$ (defined as individual frequency-dependent 1PDMs on the right-hand side of Eqs. (22) and (24)), which are formed either between zero-order and second-order response wave functions or between two first-order response wave functions. These individual $\omega \mathrm{DMs}$ thus represent the transitions between zeroorder and second-order states and between two first-order states, respectively, even though the overall transition is back to the initial state.

The second-order response states $X_{\varepsilon,-\omega_{a} ; \varepsilon, \omega_{c}}^{b c}$ and $\tilde{X}_{\varepsilon, \omega_{c} ; \varepsilon,-\omega_{a}}^{c b}$ in Eq. (22) have $b c$ symmetry. In addition, the states can be attributed psuedo-energies $-\omega_{a}$ and $\omega_{a}$ relative to the initial zero-order state $\Psi$ on the basis of the second-order response equations, Eqs. (14) and (15), involving $-\omega_{a}$. Thus, the $\omega \mathrm{DM}$ between $\Psi$ and $X_{\varepsilon,-\omega_{a} ; \varepsilon, \omega_{c}}^{b c}$ represents the contribution from the coupling between the initial zero-order state and a second-order response state that is $-\omega_{a}$ above the initial zero-order state. Similarly, the $\omega \mathrm{DM}$ between $\Psi$ and $\tilde{X}_{\varepsilon, \omega_{c} ; \varepsilon,-\omega_{a}}$ represents the contribution from the coupling between the initial zero-order state and a second-order response state that is $-\omega_{a}$ below the initial state. The first-order response states $\tilde{X}_{\varepsilon, \omega_{b}}^{b}$ and $X_{\varepsilon, \omega_{c}}^{c}$ represent response states that are $\omega_{b}$ below and $\omega_{c}$ above the initial zero-order state. The $\omega \mathrm{DM}$ s between these states again represents the contribution from the coupling between the states that are $-\omega_{a}=\omega_{b}+\omega_{c}$ apart. Combining these $\omega \mathrm{DMs}, \gamma_{\varepsilon, \omega_{1}, \omega_{2}}^{b c}$ therefore carries the contribution of coupling between states that are separated by the energy of the sum-frequency photon. In addition, we also note that when the initial state is the ground state, the contribution from $\omega \mathrm{DMs}$ between two first-order response states to an SFG process would not be dominant due to near-resonance consideration. However, these $\omega \mathrm{DMs}$ can be dominant for excited-state response 1PDMs. $\gamma_{\varepsilon, \omega_{1}, \omega_{2}}^{b c}$ also has the same symmetry as the two-photon virtual state.

The second-order response states $X_{-\varepsilon,-\omega_{b} ; \varepsilon, \omega_{c}}^{a c}$ and $\tilde{X}_{\varepsilon, \omega_{c} ;-\varepsilon,-\omega_{b}}^{c a}$ in Eq. (24) have $a c$ symmetry, and have psuedoenergies of $-\omega_{b}$ and $\omega_{b}$ relative to the initial zero-order state. Similarly, the first-order response states $\tilde{X}_{-\varepsilon, \omega_{a}}^{a}$ and $X_{\varepsilon, \omega_{c}}^{c}$ also differ by $-\omega_{a}-\omega_{c}=\omega_{b}$. Therefore, $\tilde{\gamma}_{\varepsilon, \omega_{1}, \omega_{2}}^{a c}$ carries the contribution of coupling between states that are separated by the energy $\omega_{b}$ of an absorbed photon. $\tilde{\gamma}_{\varepsilon, \omega_{1}, \omega_{2}}^{a c}$ also has the same symmetry as the one-photon virtual state.

The SFG process can also be interpreted as a transition between the two-photon virtual state and the zero-order initial state, as shown in Fig. 1. $\beta$ in terms of the transition 1PDM for this "virtual" transition would also be given by Eq. (21). Thus, the response 1PDM in Eq. (22) can also be interpreted as the transition 1PDM between these states. In analogy of Eq. (6), the two-photon virtual state $\left(\Psi_{2 \mathrm{P}-\text { virtual }}\right)$ is given by $\Psi_{2 \mathrm{P} \text {-virtual }, \varepsilon, \omega_{1}, \omega_{2}}^{b c}=\sum_{p q}\left[\gamma_{\varepsilon, \omega_{1}, \omega_{2}}^{b c}\right]_{p q} p^{\dagger} q\left|\Psi_{k}\right\rangle+$ higher excitations.
The transition to the two-photon virtual state can be characterized using the corresponding complex-valued exciton's wave function given by

$$
\Psi_{2 \mathrm{P}-\mathrm{exc}, \varepsilon, \omega_{1}, \omega_{2}}^{b c}\left(r_{h}, r_{e}\right)=\sum_{p q}\left[\gamma_{\varepsilon, \omega_{1}, \omega_{2}}^{b c}\right]_{p q} \phi_{p}\left(r_{e}\right) \phi_{q}\left(r_{h}\right) .
$$

Similarly, the one-photon virtual state can be expressed using response 1PDMs in Eqs. (23) and (24) according to

$\Psi_{1 \mathrm{P}-\text { virtual }, \varepsilon, \omega_{1}, \omega_{2}}^{a c}=\sum_{p q}\left[\tilde{\gamma}_{\varepsilon, \omega_{1}, \omega_{2}}^{a c}\right]_{p q} p^{\dagger} q\left|\Psi_{k}\right\rangle+$ higher excitations.

The transition to the one-photon virtual state can be characterized using the corresponding complex-valued exciton's wave function given by

$$
\Psi_{1 \mathrm{P}-\mathrm{exc}, \varepsilon, \omega_{1}, \omega_{2}}^{a c}\left(r_{h}, r_{e}\right)=\sum_{p q}\left[\tilde{\gamma}_{\varepsilon, \omega_{1}, \omega_{2}}^{a c}\right]_{p q} \phi_{p}\left(r_{e}\right) \phi_{q}\left(r_{h}\right) .
$$

Below, we call both $\gamma_{\varepsilon, \omega_{1}, \omega_{2}}^{b c}$ and $\tilde{\gamma}_{\varepsilon, \omega_{1}, \omega_{2}}^{b c}$ in Eqs. (22) and (24) as a general second-order response 1PDM $\gamma_{\omega_{1}, \omega_{2}}^{b c}$ and drop the index $\varepsilon$ for brevity. We also label $\Psi_{1 \mathrm{P} \text {-exc, } \varepsilon, \omega_{1}, \omega_{2}}^{b c}$ and $\Psi_{2 \mathrm{P} \text {-exc, } \varepsilon, \omega_{1}, \omega_{2}}^{b c}$ in Eqs. (28) and (26) as a general $\Psi_{\mathrm{exc}, \omega_{1}, \omega_{2}}^{b c}$ such that

$$
\Psi_{\mathrm{exc}, \omega_{1}, \omega_{2}}^{b c}\left(r_{h}, r_{e}\right)=\sum_{p q}\left[\gamma_{\omega_{1}, \omega_{2}}^{b c}\right]_{p q} \phi_{p}\left(r_{e}\right) \phi_{q}\left(r_{h}\right) .
$$

The exciton's wave function related to the overall transition underlying $\beta$ can be expressed using $\Psi_{\mathrm{exc}, \omega_{1}, \omega_{2}} \mathrm{~S}$ as follows:

$$
\Psi_{\mathrm{exc}, \omega_{1}, \omega_{2}}\left(r_{h}, r_{e}\right)=\sum_{b, c \in\{x, y, z\}} \Psi_{\mathrm{exc}, \omega_{1}, \omega_{2}}^{b c}\left(r_{h}, r_{e}\right) \hat{b} \hat{c} .
$$

Compared to the one-component $\Psi_{\text {exc }}$ in Eq. (7) for a onephoton transition between two zero-order electronic states, the exciton's wave function in Eq. (30) has $3 \times 3$ components. This wave function maps the changes in the spatial electronic distribution due to the interaction with photons onto characteristic orbitals and can provide physically meaningful quantities such as the average distance between the electron and hole distributions $\left(d_{h \rightarrow e}\right)$, the size of the exciton $\left(d_{\mathrm{exc}}\right)$, and the degree of charge transfer $(\mathcal{Q})$ from one part of the system to another (or the probability of finding the hole on one fragment and the electron on the other ${ }^{29,30}$. For example, $d_{\mathrm{exc}}$, which quantifies the degree of dynamic charge transfer (or charge resonance), is expressed as follows:

$$
\begin{aligned}
d_{\mathrm{exc}, \omega_{1}, \omega_{2}} & =\sqrt{\left\langle\Psi_{\mathrm{exc}, \omega_{1}, \omega_{2}}\left|\left(r_{h}-r_{e}\right)^{2}\right| \Psi_{\left.\mathrm{exc}, \omega_{1}, \omega_{2}\right\rangle}\right\rangle} \\
& =\sqrt{\frac{\sum_{b c}\left\|\gamma_{\omega_{1}, \omega_{2}}^{b c}\right\|^{2}\left(d_{\mathrm{exc}, \omega_{1}, \omega_{2}}^{b c}\right)^{2}}{\left.\sum_{b c}\left\|\gamma_{\omega_{1}, \omega_{2}}^{b c}\right\|\right|^{2}}}
\end{aligned}
$$

with

$$
\begin{aligned}
& d_{\mathrm{exc}, \omega_{1}, \omega_{2}}^{b c}=\sqrt{\left\langle\Psi_{\mathrm{exc}, \omega_{1}, \omega_{2}}^{b c}\left|\left(r_{h}-r_{e}\right)^{2}\right| \Psi_{\mathrm{exc}, \omega_{1}, \omega_{2}}^{b c}\right\rangle} \\
& =\sqrt{\frac{\left\|\gamma_{\omega_{1}, \omega_{2}}^{b c, \mathrm{Re}}||^{2}\left(d_{\mathrm{exc}, \omega_{1}, \omega_{2}}^{b c, \operatorname{Re}}\right)^{2}+\right\| \gamma_{\omega_{1}, \omega_{2}}^{b c, \operatorname{Im}} \|^{2}\left(d_{\mathrm{exc}, \omega_{1}, \omega_{2}}^{b c, \mathrm{Im}}\right)^{2}}{\left\|\gamma_{\omega_{1}, \omega_{2}}^{b c, \operatorname{Re}}\right\|^{2}+\left\|\gamma_{\omega_{1}, \omega_{2}}^{c c, \operatorname{Im}}\right\|^{2}}},
\end{aligned}
$$




$$
d_{\mathrm{exc}, \omega_{1}, \omega_{2}}^{b c, \mathrm{Re} / \mathrm{Im}}=\sqrt{\left\langle\Psi_{\mathrm{exc}, \omega_{1}, \omega_{2}}^{b c, \mathrm{Re} / \mathrm{Im}}\left|\left(r_{h}-r_{e}\right)^{2}\right| \Psi_{\mathrm{exc}, \omega_{1}, \omega_{2}}^{b c, \mathrm{Re} / \mathrm{Im}}\right.},
$$

and

$$
\left\|\gamma_{\omega_{1}, \omega_{2}}^{b c}\right\|^{2}=\left\|\gamma_{\omega_{1}, \omega_{2}}^{b c, \operatorname{Re}}\right\|^{2}+\left\|\gamma_{\omega_{1}, \omega_{2}}^{b c, \operatorname{Im}}\right\|^{2}
$$

$d_{h \rightarrow e}$, on the other hand, quantifies the permanent charge transfer and is given by

$$
\begin{aligned}
d_{h \rightarrow e, \omega_{1}, \omega_{2}} & =\|\left\langle\Psi_{\mathrm{exc}, \omega_{1}, \omega_{2}}\left|r_{e}\right| \Psi_{\mathrm{exc}, \omega_{1}, \omega_{2}}\right\rangle \\
& -\left\langle\Psi_{\mathrm{exc}, \omega_{1}, \omega_{2}}\left|r_{h}\right| \Psi_{\left.\mathrm{exc}, \omega_{1}, \omega_{2}\right\rangle \|}\right\rangle \\
& =\left\|\left\langle r_{e}\right\rangle \omega_{1}, \omega_{2}-\left\langle r_{h}\right\rangle \omega_{1}, \omega_{2}\right\|,
\end{aligned}
$$

where the Cartesian components of $\left\langle r_{e}\right\rangle \omega_{1}, \omega_{2}-\left\langle r_{h}\right\rangle_{\omega_{1}, \omega_{2}}$ are given by

$$
\begin{gathered}
\left\langle z_{e}\right\rangle \omega_{1}, \omega_{2}-\left\langle z_{h}\right\rangle \omega_{1}, \omega_{2} \\
=\frac{\sum_{b c}\left\|\gamma_{\omega_{1}, \omega_{2}}^{b c, \operatorname{Re}}\right\|^{2}\left(\left\langle z_{e}\right\rangle_{\omega_{1}, \omega_{2}}^{b c, \operatorname{Re}}-\left\langle z_{h}\right\rangle_{\omega_{1}, \omega_{2}}^{b c, \operatorname{Re}}\right)}{\sum_{b c}\left\|\gamma_{\omega_{1}, \omega_{2}}^{b c}\right\|^{2}} \\
+\frac{\sum_{b c}\left\|\gamma_{\omega_{1}, \omega_{2}}^{b c, I m}\right\|^{2}\left(\left\langle z_{e}\right\rangle_{\omega_{1}, \omega_{2}}^{b c, \operatorname{Im}}-\left\langle z_{h}\right\rangle_{\omega_{1}, \omega_{2}}^{b c, I m}\right)}{\sum_{b c}\left\|\gamma_{\omega_{1}, \omega_{2}}^{b c}\right\|^{2}}
\end{gathered}
$$

and

$$
\left\langle z_{h / e}\right\rangle_{\omega_{1}, \omega_{2}}^{b c, \operatorname{Re} / \mathrm{Im}}=\|\left\langle\Psi_{\mathrm{exc}, \omega_{1}, \omega_{2}}^{b c, \mathrm{Re} / \mathrm{Im}}\left|z_{h / e}\right| \Psi_{\mathrm{exc}, \omega_{1}, \omega_{2}}^{b c, \operatorname{Re} / \mathrm{Im}}\right\rangle .
$$

The inter-fragment charge transfer between two fragments of the system (say, $A$ and $B$ ) is given according to

$$
\begin{aligned}
& \mathcal{Q}_{\omega_{1}, \omega_{2}}= \\
& \frac{\sum_{b c} \sum_{v \in A, \rho \in B}\left(\left[\gamma_{\omega_{1}, \omega_{2}}^{b c, \operatorname{Re}}\right]_{v \rho}^{2}+\left[\gamma_{\omega_{1}, \omega_{2}}^{b c, \operatorname{Im}}\right]_{v \rho}^{2}\right)}{\sum_{b c}\left\|\gamma_{\omega_{1}, \omega_{2}}^{b c}\right\|^{2}} \\
& +\frac{\sum_{b c} \sum_{v \in B, \rho \in A}\left(\left[\gamma_{\omega_{1}, \omega_{2}}^{b c, \operatorname{Re}}\right]_{v \rho}^{2}+\left[\gamma_{\omega_{1}, \omega_{2}}^{b c, \operatorname{Im}}\right]_{v \rho}^{2}\right)}{\sum_{b c}\left\|\gamma_{\omega_{1}, \omega_{2}}^{b c}\right\|^{2}} .
\end{aligned}
$$

In analogy to the exciton's wavefunction for one-photon transitions, the most compact representation of the complexvalued exciton's wavefunction in Eq. (29) is in terms of NOs ${ }^{29-36}$. Following the same steps as in Eqs. (8)-(10), we can write

$$
\begin{gathered}
\Psi_{\mathrm{exc}, \omega_{1}, \omega_{2}}^{b c}\left(r_{h}, r_{e}\right)=\sum_{S}\left(\sigma_{\omega_{1}, \omega_{2}}^{b c, \operatorname{Re}}\right)_{S} \psi_{\omega_{1}, \omega_{2}, S}^{b c, e, \operatorname{Re}}\left(r_{e}\right) \psi_{\omega_{1}, \omega_{2}, S}^{b c, h, \operatorname{Re}}\left(r_{h}\right) \\
+i \sum_{S}\left(\sigma_{\omega_{1}, \omega_{2}}^{b c, \operatorname{Im}}\right)_{S} \psi_{\omega_{1}, \omega_{2}, S}^{b c, e, \operatorname{Im}}\left(r_{e}\right) \psi_{\omega_{1}, \omega_{2}, S}^{b c, h, \operatorname{Im}}\left(r_{h}\right)
\end{gathered}
$$

The sum of the squares of the singular values equals the Frobenius norm of corresponding $\gamma$ as follows:

$$
\left\|\gamma_{\omega_{1}, \omega_{2}}^{b c, \operatorname{Re} / \mathrm{Im}}\right\|^{2}=\sum_{p q}\left(\gamma_{\omega_{1}, \omega_{2}}^{b c, \mathrm{Re} / \mathrm{Im}}\right)_{p q}^{2}=\sum_{S}\left(\sigma_{\omega_{1}, \omega_{2}}^{b c, \mathrm{Re} / \mathrm{Im}}\right)_{S}^{2} .
$$

so that singular values relate to the weights of different NO pairs to the respective real or imaginary electronic density and the exciton's wave function. In the discussion that follows, the singular values correspond to the real and imaginary components of the normalized $\left\|\gamma^{b c}\right\|$.

In contrast to the zero-order 1PDMs for which the singular values are non-negative, the response 1PDMs, $\gamma_{\omega_{1}, \omega_{2}}^{b c, R e}$ and $\gamma_{\omega_{1}, \omega_{2}}^{b c, I m}$, are traceless and no longer positive semi-definite. Whereas the singular values of zero-order 1PDMs correspond to the occupancy numbers of the NOs, the singular values of second-order response 1PDMs can be interpreted as secondorder changes in the occupancy numbers if the zero-order and second-order NOs are (approximately) the same. The orbital transitions between the hole and particle NOs for the response 1PDMs have the symmetry of the $b c$ irreducible representation. When the Cartesian indices $b$ and $c$ fall under different irreducible representations, the hole and particle NOs for a given singular value is different by symmetry. The pairs of NOs corresponding to the largest singular values contribute the largest to the second-order changes in the electronic distribution in the presence of external perturbations and provide the orbital characteristics of the underlying multiphoton process. These response NOs facilitate the visualization of the underlying multiphoton process of $\beta$.

The real component of $\beta^{a b c}$ accumulates the doubly offresonance and doubly near-resonance contributions from individual SOS terms (Fig. 2); therefore, the real component of the corresponding response 1PDM contains the cumulative orbital information of these doubly off-resonance and doubly near-resonance contributions. Similarly, since the imaginary component of $\beta^{a b c}$ accumulates the contributions from SOS terms for which one of the two photons is nearly resonant and the other is off resonant, the imaginary component of the corresponding response 1PDM contains the cumulative orbital information of these singly near-resonance contributions. The response NOs, obtained separately from these real and imaginary response 1PDMs, comprise two distinct regimes of contributing orbital channels that facilitate the change in the electronic density distribution in the course of the underlying multiphoton process.

The relative significance of the doubly off-resonance plus the doubly near-resonance contributions and the singly nearresonance contributions to a given $\beta^{a b c}$ component can be quantified from the norms $\left\|\gamma^{b c, \operatorname{Re}}\right\|$ and $\left\|\gamma^{b c, \operatorname{Im}}\right\|$ of the corresponding real and imaginary response 1 PDMs, respectively. For example, if $v^{b c, \operatorname{Im}}=\frac{\left\|\gamma^{b c, \operatorname{Im}}\right\|^{2}}{\left\|\gamma^{b c}\right\|^{2}} \approx 1$ or $v^{b c, \operatorname{Re}}=\frac{\left\|\gamma^{b c, \operatorname{Re}}\right\|^{2}}{\left\|\gamma^{b c}\right\|^{2}} \approx 0$, the corresponding $b c$ component of the exciton's wave function can be approximated by just the singly near-resonance contribution. The cumulative significance of all singly or doubly near-resonance channels to $\beta$ can be quantified from the relative norms of all nine response 1PDMs. For example, if $\Upsilon^{\mathrm{Im}}=\sum_{b c} \Upsilon^{b c, \operatorname{Im}} \approx 1$ or $\Upsilon^{\mathrm{Re}}=\sum_{b c} \Upsilon^{b c, \text { Re }} \approx 0$, where $\Upsilon^{b c, \operatorname{Im}}=\frac{\left\|\gamma^{b c, \operatorname{Im}}\right\|^{2}}{\sum_{b c}\left\|\gamma^{b c}\right\|^{2}}$ and $\Upsilon^{b c, \operatorname{Re}}=\frac{\left\|\gamma^{b c, \operatorname{Re}}\right\|^{2}}{\sum_{b c}\left\|\gamma^{b c}\right\|^{2}}$, then the response of the electronic density underlying the multiphoton process of $\beta$ is dominated by the singly near-resonance orbital channels for the given choice of photon energies. 


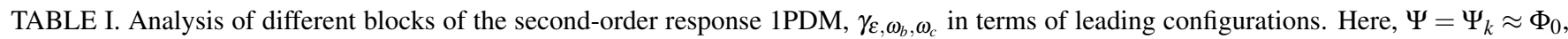
$\Psi_{m} \approx \Phi_{i}^{d}$, and $\Psi_{n} \approx \Phi_{j}^{e}$, " $\delta$ " is the Kronecker delta, $\mathcal{E}$ s are the orbital energies, and $s$ is the sign enforced by the operator $\frac{1}{2} \mathcal{C}_{ \pm \omega}$. Note that $\omega^{\prime}$ and $\omega^{\prime \prime}$ can be the energies of absorbed photons or the sum-frequency photon.

\begin{tabular}{|c|c|c|c|}
\hline$\omega \mathrm{DM}$ & $\omega \mathrm{DM}$ as SOS & $\begin{array}{l}\gamma_{\varepsilon}^{X Y, s} \\
\text { block }\end{array}$ & Coefficient \\
\hline \multirow{4}{*}{$\left\langle\Psi\left|p^{\dagger} q\right| X_{-\varepsilon^{\prime},-\omega^{\prime} ; \varepsilon^{\prime \prime}, \omega^{\prime \prime}}^{X Y}\right\rangle$} & $\left\langle\Phi_{0}\left|p^{\dagger} q\right| \Phi_{i}^{d}\right\rangle\left\langle\Phi_{i}^{d}\left|\overline{\hat{\mu}}^{X}\right| \Phi_{j}^{e}\right\rangle\left\langle\Phi_{j}^{e}\left|\hat{\mu}^{Y}\right| \Phi_{0}\right\rangle$ & \multirow[t]{2}{*}{$O V$} & \multirow{2}{*}{$\delta_{d e} \frac{\left\langle i\left|\overline{\hat{\mu}}^{X}\right| j\right\rangle\left\langle j\left|\hat{\mu}^{Y}\right| d\right\rangle}{\left(\mathcal{E}_{d}-\mathcal{E}_{i}+s \omega^{\prime}+i \mathcal{E}^{\prime}\right)\left(\mathcal{E}_{d}-\mathcal{E}_{j}-s \omega^{\prime \prime}-i \varepsilon^{\prime \prime}\right)}$} \\
\hline & $\overline{\left(\mathcal{E}_{d}-\mathcal{E}_{i}+s \omega^{\prime}+i \varepsilon^{\prime}\right)\left(\mathcal{E}_{e}-\mathcal{E}_{j}-s \omega^{\prime \prime}-i \varepsilon^{\prime \prime}\right)}$ & & \\
\hline & & \multirow[t]{2}{*}{$O V$} & $\left\langle d\left|\overline{\hat{\mu}}^{X}\right| e\right\rangle\left\langle e\left|\hat{\mu}^{Y}\right| i\right\rangle$ \\
\hline & & & $\delta_{i j} \overline{\left(\mathcal{E}_{d}-\mathcal{E}_{i}+s \omega^{\prime}+i \varepsilon^{\prime}\right)\left(\mathcal{E}_{e}-\mathcal{E}_{i}-s \omega^{\prime \prime}-i \varepsilon^{\prime \prime}\right)}$ \\
\hline \multirow{4}{*}{$\left\langle\tilde{X}_{\mathcal{\varepsilon}^{\prime}, \omega^{\prime}}^{X}\left|p^{\dagger} q\right| X_{\mathcal{\varepsilon}^{\prime \prime}, \omega^{\prime \prime}}^{Y}\right\rangle$} & $\left\langle\Phi_{0}\left|\hat{\mu}^{X}\right| \Phi_{i}^{d}\right\rangle\left\langle\Phi_{i}^{d}\left|p^{\dagger} q\right| \Phi_{j}^{e}\right\rangle\left\langle\Phi_{j}^{e}\left|\hat{\mu}^{Y}\right| \Phi_{0}\right\rangle$ & \multirow[t]{2}{*}{$O O$} & \multirow{2}{*}{$\delta_{d e} \frac{\left\langle d\left|\hat{\mu}^{X}\right| i\right\rangle\left\langle j\left|\hat{\mu}^{Y}\right| d\right\rangle}{\left(\mathcal{E}_{d}-\mathcal{E}_{i}+s \omega^{\prime}+i \mathcal{E}^{\prime}\right)\left(\mathcal{E}_{d}-\mathcal{E}_{j}-s \omega^{\prime \prime}-i \mathcal{E}^{\prime \prime}\right)}$} \\
\hline & $\overline{\left(\mathcal{E}_{d}-\mathcal{E}_{i}+s \omega^{\prime}+i \varepsilon^{\prime}\right)\left(\mathcal{E}_{e}-\mathcal{E}_{j}-s \omega^{\prime \prime}-i \mathcal{E}^{\prime \prime}\right)}$ & & \\
\hline & & $V V$ & $\left\langle i\left|\hat{\mu}^{X}\right| d\right\rangle\left\langle e\left|\hat{\mu}^{Y}\right| i\right\rangle$ \\
\hline & & & $\delta_{i j} \overline{\left(\mathcal{E}_{d}-\mathcal{E}_{i}+s \omega^{\prime}+i \varepsilon^{\prime}\right)\left(\mathcal{E}_{e}-\mathcal{E}_{i}-s \omega^{\prime \prime}-i \varepsilon^{\prime \prime}\right)}$ \\
\hline \multirow[t]{2}{*}{$\left\langle\tilde{X}_{\mathcal{E}^{\prime}, \omega^{\prime}}^{X} \mid X_{\mathcal{E}^{\prime \prime}, \omega^{\prime \prime}}^{Y}\right\rangle\left\langle\Psi\left|p^{\dagger} q\right| \psi\right\rangle$} & $\underline{\left\langle\Phi_{0}\left|\hat{\mu}^{X}\right| \Phi_{i}^{d}\right\rangle\left\langle\Phi_{i}^{d} \mid \Phi_{j}^{e}\right\rangle\left\langle\Phi_{j}^{e}\left|\hat{\mu}^{Y}\right| \Phi_{0}\right\rangle\left\langle\Phi_{0}\left|p^{\dagger} q\right| \Phi_{0}\right\rangle}$ & \multirow[t]{2}{*}{$O O$} & \multirow{2}{*}{$\delta_{i j} \delta_{d e} \frac{\left\langle d\left|\hat{\mu}^{X}\right| i\right\rangle\left\langle i\left|\hat{\mu}^{I}\right| d\right\rangle}{\left(\mathcal{E}_{d}-\mathcal{E}_{i}+s \omega^{\prime}+i \mathcal{E}^{\prime}\right)\left(\mathcal{E}_{d}-\mathcal{E}_{i}-s \omega^{\prime \prime}-i \mathcal{E}^{\prime \prime}\right)}$} \\
\hline & $\overline{\left(\mathcal{E}_{d}-\mathcal{E}_{i}+s \omega^{\prime}+i \varepsilon^{\prime}\right)\left(\mathcal{E}_{e}-\mathcal{E}_{j}-s \omega^{\prime \prime}-i \varepsilon^{\prime \prime}\right)}$ & & \\
\hline \multirow{4}{*}{$\left\langle\tilde{X}_{\mathcal{\varepsilon}^{\prime \prime}, \omega^{\prime \prime} ;-\varepsilon^{\prime},-\omega^{\prime}}^{Y X}\left|p^{\dagger} q\right| \Psi\right\rangle$} & $\left\langle\Phi_{0}\left|\hat{\mu}^{Y}\right| \Phi_{i}^{d}\right\rangle\left\langle\Phi_{i}^{d}\left|\overline{\hat{\mu}}^{X}\right| \Phi_{j}^{e}\right\rangle\left\langle\Phi_{j}^{e}\left|p^{\dagger} q\right| \Phi_{0}\right\rangle$ & \multirow[t]{2}{*}{$V O$} & \multirow{2}{*}{$\delta_{d e} \frac{\left\langle d\left|\hat{\mu}^{Y}\right| i\right\rangle\left\langle i\left|\overline{\hat{\mu}}^{X}\right| j\right\rangle}{\left(\mathcal{E}_{d}-\mathcal{E}_{i}+s \omega^{\prime \prime}+i \varepsilon^{\prime \prime}\right)\left(\mathcal{E}_{d}-\mathcal{E}_{j}-s \omega^{\prime}-i \varepsilon^{\prime}\right)}$} \\
\hline & $\overline{\left(\mathcal{E}_{d}-\mathcal{E}_{i}+s \omega^{\prime \prime}+i \varepsilon^{\prime \prime}\right)\left(\mathcal{E}_{e}-\mathcal{E}_{j}-s \omega^{\prime}-i \varepsilon^{\prime}\right)}$ & & \\
\hline & & \multirow[t]{2}{*}{$V O$} & 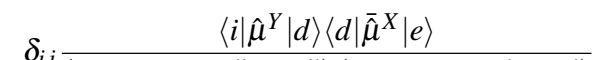 \\
\hline & & & $o_{i j} \overline{\left(\mathcal{E}_{d}-\mathcal{E}_{i}+s \omega^{\prime \prime}+i \varepsilon^{\prime \prime}\right)\left(\mathcal{E}_{e}-\mathcal{E}_{i}-s \omega^{\prime}-i \varepsilon^{\prime}\right)}$ \\
\hline
\end{tabular}

\section{Analysis of response 1PDMs in terms of leading configurations}

Next, in order to interpret the NOs obtained from the Eqs. (22) and (24), we present the configurational analysis of these response 1PDMs using a simple uncorrelated picture (i.e., within the configuration interaction singles ansatz). Table I summarizes the contribution of different $\omega \mathrm{DMs}$, present in the signed second-order response 1PDMs $\gamma_{\omega_{1}, \omega_{2}}^{b c,+}$ and $\gamma_{\omega_{1}, \omega_{2}}^{b c,-}$, to the different blocks of $\gamma_{\omega_{1}, \omega_{2}}^{b c}$. Here, we neglect correlation effects and consider only the dominant electronic configurations in the many-body electronic states such that $\Psi_{k} \approx \Phi_{0}, \Psi_{m} \approx \Phi_{i}^{d}$, and $\Psi_{n} \approx \Phi_{j}^{e}$, where $i, j$ are the occupied $(O)$ and $d, e$ are the unoccupied $(V)$ orbitals. Notably, different $\omega \mathrm{DMs}$ contribute to different blocks of the response 1PDM; some contribute exclusively to the $O O$ and $V V$ blocks and some contribute either to the $O V$ block or the $V O$ block. In the context of NOs obtained from $\gamma_{\omega_{1}, \omega_{2}}^{b c,+}$ or $\gamma_{\omega_{1}, \omega_{2}}^{b c,-}$, if the $\omega$ DMs that contribute to the $O V$ or $V O$ blocks are dominant (in terms of norm), SVD gives pairs of NOs in which one NO represents an occupied and another an unoccupied orbital. In contrast, if the $\omega \mathrm{DMs}$ that contribute to the $O O$ and $V V$ blocks are dominant, the NO pairs comprise either both occupied or both unoccupied orbitals - the hole and particle NOs can also be the same orbital if allowed by symmetry.

From Table I, for $s=+$, the two possible coefficients (weights) for the first term can be large by resonance enhance- ment when $\omega^{\prime \prime} \approx \mathcal{E}_{e}-\mathcal{E}_{j}$ is one of the absorbed photon's energy and/or $\omega^{\prime} \approx \mathcal{E}_{d}-\mathcal{E}_{i}$ is the energy of the sum-frequency photon. The two possible coefficients of the third term can be large by resonance enhancement when $\omega^{\prime} \approx \mathcal{E}_{e}-\mathcal{E}_{j}$ is one of the absorbed photon's energy and/or $\omega^{\prime \prime} \approx \mathcal{E}_{d}-\mathcal{E}_{i}$ is the energy of the sum-frequency photon. The coefficients for the second term can be large by resonance enhancement only when $\omega^{\prime \prime} \approx \mathcal{E}_{e}-\mathcal{E}_{j}$ is one of the absorbed photon's energy and/or $\omega^{\prime} \approx \mathcal{E}_{d}-\mathcal{E}_{i}$ is the energy of the sum-frequency photon. One can similarly identify the cases for which these coefficients will be large for $s=-$.

Finally, we also consider the angle $(\theta)$ between the $\gamma_{\omega_{1}, \omega_{2}}^{b c,+}$ and $\gamma_{\omega_{1}, \omega_{2}}^{b c}$ 1PDMs computed using the equation:

$$
\theta=\cos ^{-1}\left(\frac{\left\|\gamma^{b c}\right\|^{2}-\left\|\gamma^{b c,+}\right\|^{2}-\left\|\gamma^{b c,-}\right\|^{2}}{2\left\|\gamma^{b c,+}\right\|\left\|\gamma^{b c,-}\right\|}\right) .
$$

When these signed response 1PDMs only have nonzero $O \mathrm{~V}$ or $V O$ blocks and are complementary to each other, then the dot product between these signed 1PDMs is zero. In this case, $\theta=90^{\circ}$. Thus, the deviation from $90^{\circ}$ indicates the extent to which the $\omega \mathrm{DM}$ s contribute to other blocks of these signed response 1PDMs. For the static case, this angle is $0^{\circ}$ as $\gamma_{\omega_{1}, \omega_{2}}^{b,+}$ and $\gamma_{\omega_{1}, \omega_{2}}^{b c-}$ are equivalent.

For Hermitian methods, the $\gamma_{\omega_{1}, \omega_{2}}^{b c,+}$ and $\gamma_{\omega_{1}, \omega_{2}}^{b c,-}$ are transpose matrices of each other; so, the operator $\frac{1}{2} \mathcal{C}_{ \pm \omega}$ is superfluous. These signed response 1PDMs, therefore, provide the same 
NOs (only the hole and particle NOs are swapped) and other wave-function analysis metrics. Although for non-Hermitian methods such as coupled-cluster methods, $\gamma_{\omega_{1}, \omega_{2}}^{b c,+}$ and $\gamma_{\omega_{1}, \omega_{2}}^{b c,-}$ are not transpose matrices of each other, they also yield similar NOs and other wave-function analysis metrics. We illustrate this in Section IV A 1. Since there are two virtual states involved in the underlying process of $\beta$, we require both sets of response 1PDMs given by Eqs. (22) and (24) - this increases the number of response equations to be solved to construct $\gamma_{\omega_{1}, \omega_{2}}^{p c,+}$ and $\gamma_{\omega_{1}, \omega_{2}}^{p c,-}$ for Hermitian methods. For nonHermitian methods, to minimize this number, we construct $\beta^{a b c}$ according to

$\beta^{a b c}\left(\omega_{a} ; \omega_{b}, \omega_{c} ; \varepsilon\right)=\sum_{p q} \hat{\mu}_{p q}^{a}\left[\gamma_{\varepsilon, \omega_{b}, \omega_{c}}^{b c,+}\right]_{p q}+\sum_{p q} \hat{\mu}_{p q}^{b}\left[\tilde{\gamma}_{\varepsilon, \omega_{b}, \omega_{c}}^{a c,-}\right]_{p q}$

we would obtain complementary orbital information from the corresponding signed response $1 \mathrm{PDMs} \gamma_{\omega_{1}, \omega_{2}}^{b c,}$ and $\tilde{\gamma}_{\omega_{1}, \omega_{2}}^{a c,}$. In short, we can now combine the orbital analysis from the two sets of signed response orbitals obtained one each from Eqs. (22) and (24) by computing the same number of response wave functions as before. However, we cannot now construct a single response $1 \mathrm{PDM}\left(\gamma_{\omega_{1}, \omega_{2}}^{b c}\right.$ and/or $\left.\tilde{\gamma}_{\omega_{1}, \omega_{2}}^{a c}\right)$ without solving more response equations. We illustrate below how this strategy allows the construction of the full orbital picture of the SFG process and characterize the one- and two-photon virtual states in terms of orbitals and quantities such as $d_{\mathrm{exc}}^{+}, d_{h \rightarrow e}^{+}$, $\mathcal{Q}^{+}$and $\tilde{d}_{\mathrm{exc}}^{-}, \tilde{d}_{h \rightarrow e}^{-}, \tilde{\mathcal{Q}}^{-}$corresponding to $\gamma_{\omega_{1}, \omega_{2}}^{b c}$ and $\tilde{\gamma}_{\omega_{1}, \omega_{2}}^{a c}$, respectively.

\section{COMPUTATIONAL DETAILS}

We implemented the calculations of response 1PDMs corresponding to the $\beta$ tensor in a developer's version of the Q-Chem package ${ }^{43,44}$. This implementation employs the existing infrastructure of the libwfa library for wave-function analysis $\mathrm{s}^{30,34,36}$. In the exemplary calculations, we use the coupled-cluster singles and doubles (CCSD) level of theory ${ }^{42,50-52}$ and compute the $\beta$ tensor and its damped response 1PDMs within the expectation-value framework $^{47-49,53-58}$ using this new feature.

We used the CCSD/aug-cc-pVDZ optimized geometry of cis-difluoroethene. The geometries of para-nitroaniline and the hemibonded $\mathrm{OH}+\mathrm{H}_{2} \mathrm{O}$ complex were obtained from Refs. 57 and 6, respectively. The Cartesian coordinates for these systems are provided in the Supplementary Material. Q-Chem's symmetry notations are used throughout this paper; its detailed description is provided at http://iopenshell.usc.edu/resources/howto/symmetry/ and in Ref. 26. The $C_{2 v}$ symmetry group is used for cisdifluoroethene and para-nitroaniline in all our calculations. The hemibonded $\mathrm{OH}+\mathrm{H}_{2} \mathrm{O}$ complex has $C_{1}$ symmetry.

In the response calculations, we used the restricted HartreeFock (RHF) reference for cis-difluoroethene and paranitroaniline. For the hemibonded $\mathrm{OH}+\mathrm{H}_{2} \mathrm{O}$ complex, we used the unrestricted HF (UHF) reference. The Dunning aug-cc-pVDZ basis set was used in all our calculations of $\beta$. The phenomenological damping parameter $\varepsilon$ was set as 0.01 a.u., 0.05 a.u., and 0.01 a.u. for cis-difluoroethene, para-nitroaniline, and the hemibonded $\mathrm{OH}+\mathrm{H}_{2} \mathrm{O}$ complex, respectively. All NOs were visualized using the Gabedit software. Isosurfaces of $0.01,0.01$, and 0.05 were used for the three systems, respectively. We used the TheoDORE ${ }^{59}$ package for computing the component charge-transfer probabilities $\mathcal{Q}^{b c, \pm, \mathrm{Re} / \mathrm{Im}}$ following the NO analyses of component response 1PDMs with Q-Chem.

\section{RESULTS AND DISCUSSION}

In this section, we refer to the response 1PDMs in Eqs. (22) and (24) as $\gamma_{\omega_{1}, \omega_{2}}^{b c}$ and $\tilde{\gamma}_{\omega_{1}, \omega_{2}}^{b c}$ with the dependence on $\varepsilon$ implicit. Symbols with $\sim$ refer to the quantities related to $\tilde{\gamma}_{\omega_{1}, \omega_{2}}^{b c}$.

\section{A. cis-Difluoroethene}

Table II reports excitation energies, oscillator strengths, and the corresponding natural transition orbitals (NTOs) for the lowest one-photon transitions. The lowest excited state has $\mathrm{B}_{2}$ symmetry and excitation energy of $6.69 \mathrm{eV}$. The oscillator strength is the largest for the $\mathrm{XA}_{1} \rightarrow 1 \mathrm{~B}_{1}$ transition with excitation energy of $8.54 \mathrm{eV}$. This transition is the $\pi-\pi^{*}$ transition, as characterized by the $b_{2}$ hole (resembling HOMO) and $a_{2}$ particle NTOs. Below, we compute the $\beta$ tensor corresponding to undamped non-resonant SFG, damped onephoton SFG, damped two-photon SFG, and doubly resonant SFG, as well as the undamped static case. We characterize the response 1PDMs associated with the dominant components of the $\beta$ tensor in terms of NOs for these different cases. The important orbital channels across these different cases are shown in Fig. 3. Below, we explain how we obtain this orbital information using our wave-function analysis toolkit.

\section{Undamped $\beta_{0}(-2 \omega ; \omega, \omega ; 0)$}

In this section, we characterize the orbitals involved in the SFG process involving degenerate photons, i.e., $\omega_{1}=\omega_{2}=$ $\omega=4.00 \mathrm{eV}$. For such a case, $\beta^{a b c}$ and $\beta^{a c b}$ are the same. Tables S1 in the Supplementary Material gives the nonzero $\beta^{a b c}$ values for these photon energies, which shows that the dominant components are $\beta^{x x z}$ and $\beta^{x z x}$ (equal by symmetry) followed by $\beta^{z y y}$.

We now characterize the response 1PDMs obtained according to Eq. (22) corresponding to the dominant $\beta$ components. For this case with degenerate $\omega_{1}$ and $\omega_{2}, \gamma_{\omega, \omega}^{b c}$ and $\gamma_{\omega, \omega}^{c b}$ are equivalent. In terms of the norms, $\gamma_{\omega, \omega}^{x y}$ and $\gamma_{\omega, \omega}^{y x}$ are dominant due to the enhancement from the near-resonance of the sum frequency and the excitation energy of the $1 \mathrm{~A}_{2}$ state. However, these response 1 PDMs do not contribute to the $\beta_{0}$ tensor by symmetry.

Since $\beta^{x x z}$ and $\beta^{x z x}$ are dominant, we now consider the corresponding $\gamma_{\omega, \omega}^{x z}$ (and, equivalently, $\gamma_{\omega, \omega}^{z x}$ ) response 1PDM., 


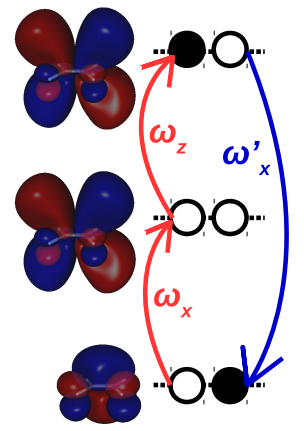

(a)

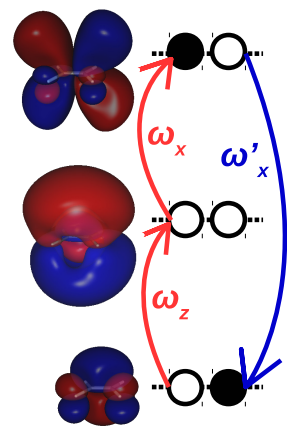

(b)

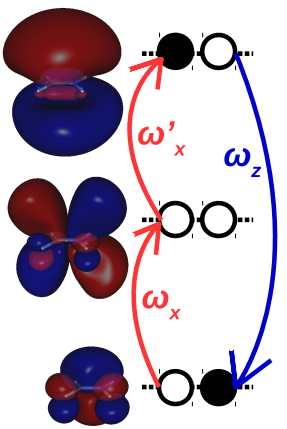

(c)

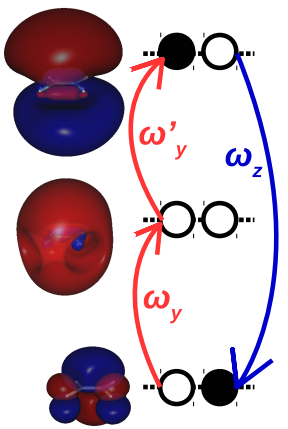

(d)

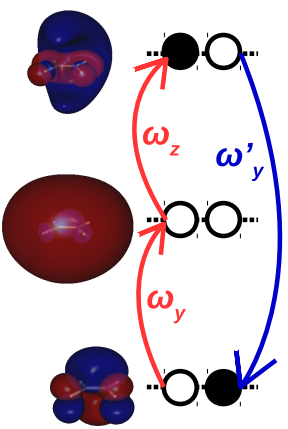

(e)

FIG. 3. The important orbital channels for $\mathrm{C}_{2} \mathrm{~F}_{2} \mathrm{H}_{2}$ in the SFG process for different sets of photon energies obtained from the NO analyses of corresponding response 1PDMs. (a) The $b_{2} \stackrel{\omega_{x}}{\longrightarrow} a_{2} \stackrel{\omega_{z}}{\longrightarrow} a_{2} \stackrel{-\omega_{x}^{\prime}}{\longrightarrow} b_{2}$ orbital channel. (b) The $b_{2} \stackrel{\omega_{z}}{\longrightarrow} \tilde{b}_{2} \stackrel{\omega_{x}}{\longrightarrow} a_{2} \stackrel{-\omega_{x}^{\prime}}{\longrightarrow} b_{2}$ orbital channel. (c) $b_{2} \stackrel{\omega_{x}}{\longrightarrow} a_{2} \stackrel{\omega_{x}^{\prime}}{\longrightarrow} \tilde{b}_{2} \stackrel{-\omega_{z}}{\longrightarrow} b_{2}$ orbital channel. (d) $b_{2} \stackrel{\omega_{y}}{\longrightarrow} \tilde{a}_{1} \stackrel{\omega_{y}^{\prime}}{\longrightarrow} \tilde{b}_{2} \stackrel{-\omega_{z}}{\longrightarrow} b_{2}$ orbital channel. (e) $b_{2} \stackrel{\omega_{y}}{\longrightarrow} a_{1} \stackrel{\omega_{z}}{\longrightarrow} \tilde{a}_{1} \stackrel{-\omega_{y}^{\prime}}{\longrightarrow} b_{2}$ orbital channel. Here, $b_{2}$ and $a_{2}$ NOs resemble the hole and particle NTOs of the $\mathrm{XA}_{1} \rightarrow 1 \mathrm{~B}_{1}$ transition, $\tilde{b}_{2}$ resembles the particle NTO of the XA $\rightarrow 2 \mathrm{~A}_{1}$ transition, $a_{1}$ resembles the particle $\mathrm{NTO}$ of the $\mathrm{XA}_{1} \rightarrow 1 \mathrm{~B}_{2}$ transition, and $\tilde{a}_{1}$ resembles a combination of particle $\mathrm{NTOs}$ of $\mathrm{XA}_{1} \rightarrow 1 \mathrm{~B}_{2}$ and $\mathrm{XA}_{1} \rightarrow$ $2 \mathrm{~B}_{2}$ transitions.

TABLE II. Excitation energies $(\Omega, \mathrm{eV})$, oscillator strengths $(f)$, permanent dipole moments ${ }^{a}(\langle\mu\rangle$, a.u.), and wave-function analysis for low-lying excited states in cis-difluoroethene. The wave-function analysis for each transition includes the norm of transition 1PDM $(\|\gamma\|)$, participation ratio $(P R)$, and dominant NTO pairs (isovalue is $0.01)$. The singular values $\left(\sigma_{K}\right)$ correspond to normalized transition 1PDMs. EOM-EE-CCSD/aug-cc-pVDZ.

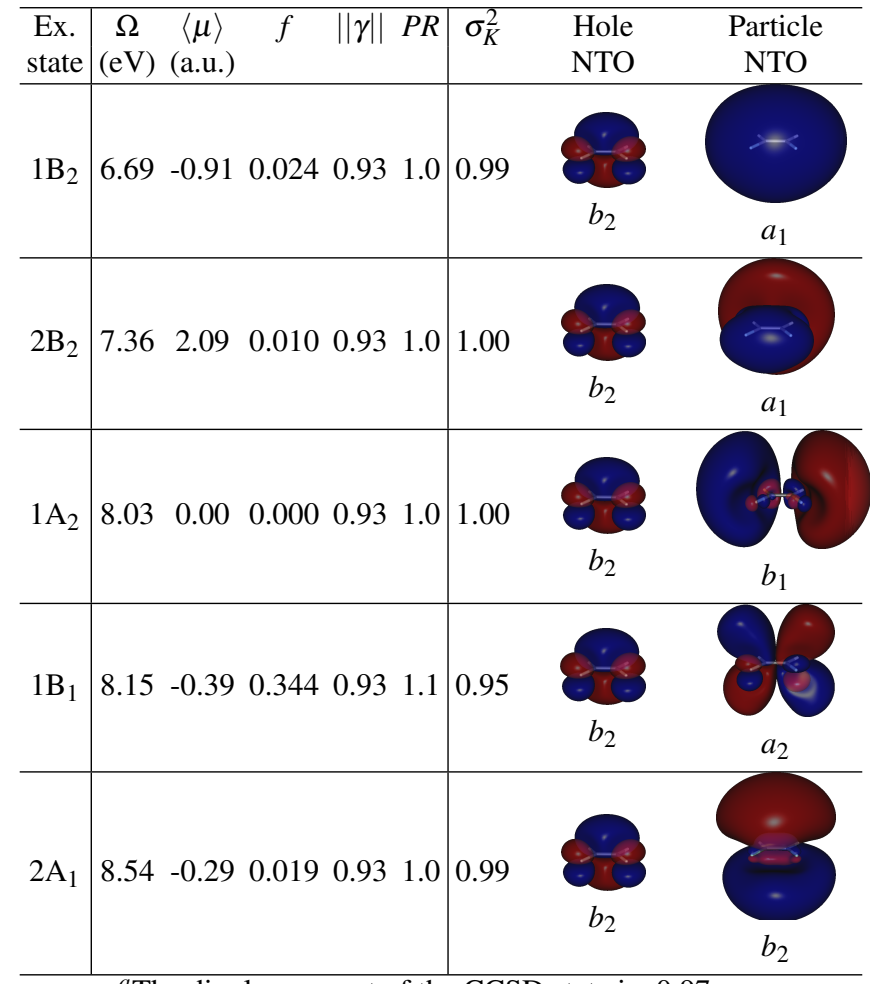

${ }^{a}$ The dipole moment of the CCSD state is -0.97 a.u.

which is characterized by two pairs of hole and particle NOs of similar singular values (see Table S2 in Supplementary Material). One of these NO pair gives a $b_{2} \rightarrow a_{2}$ orbital transi- tion similar to the NTOs computed for the $\mathrm{XA}_{1} \rightarrow 1 \mathrm{~B}_{1} \pi-$ $\pi^{*}$ transition. The component signed response 1PDM $\gamma_{\omega, \omega}^{x z,+}$ is characterized by this NO pair as shown in Table S3 of Supplementary Material. This also suggests that the $\omega \mathrm{DM}$ s that contribute to the $O V$ block of $\gamma_{\omega, \omega}^{x z,+}$ dominate, resulting in a $O \rightarrow V$ orbital transition. The second NO pair of $\gamma_{\omega, \omega}^{x z}$ is similar but with the hole and particle NOs swapped relative to the first NO pair. This arises from $\gamma_{\omega, \omega}^{b c,-}$, which is dominated by $\omega \mathrm{DMs}$ contributing to the $V O$ block.

Next, we analyze the $\gamma_{\omega, \omega}^{y y}$ response 1PDM that correspond to $\beta^{z y y}$. The NO analysis of $\gamma^{y y}$ in (see Table S2 in Supplementary Material) gives two NO pairs. The first NO pair has hole and particle NOs both given by a $b_{2}$ orbital that is a combination of the hole and particle NTOs of the $\mathrm{XA}_{1} \rightarrow$ $2 \mathrm{~A}_{1}$ transition, although resembling the hole NTO more than the particle NTO. The second NO pair has hole and particle NOs both given by a similar $b_{2}$ orbital with a more complex nodal structure; this orbital resembles the particle NTO of the $\mathrm{XA}_{1} \rightarrow 2 \mathrm{~A}_{1}$ transition more than the hole NTO). The orbital transitions associated with $\gamma_{\omega, \omega}^{y y}$ remain unclear since the same NO is present for the hole and the particle orbitals in each NO pair. This is not unexpected as $\gamma_{\omega, \omega}^{y y}$ is symmetric for Hermitian theories; but for non-Hermitian CCSD response, the hole and particle NOs can be different even if they have the same symmetry. So, we augment the NO analysis of $\gamma_{\omega, \omega}^{y y}$ with the separate NO analyses of $\gamma_{\omega, \omega}^{y y,+}$ and $\gamma_{\omega, \omega}^{y y,-}$ in Table S3 of Supplementary Material. For these signed response 1PDMs, the NO analysis gives one NO pair comprising dissimilar hole and particle $b_{2}$ NOs. The NO pair for $\gamma_{\omega, \omega}^{y y,+}$ gives a $b_{2} \rightarrow b_{2}$ orbital transition that is similar to the NTOs computed for the $\mathrm{XA}_{1} \rightarrow$ $2 \mathrm{~A}_{1}$ transition. This is a result of the dominant $O V$ block in $\gamma_{\omega, \omega}^{y y,+}$, which results in a $O \rightarrow V$ orbital transition. The NO pair for $\gamma_{\omega, \omega}^{y y,-}$ gives the reversed orbital transition, a result of the dominant $V O$ block. This added layer of analysis thus facilitates the characterization of transitions between two $b_{2}$ orbitals dominating the third-order process.

For all component $\gamma_{\omega, \omega}^{b c}$ response 1PDMs, the angle $\theta$ from 
Eq. (41) is very close to $90^{\circ}$, indicating that each pair of $\gamma_{\omega, \omega}^{b c,+}$ and $\gamma_{\omega, \omega}^{b c,-}$ have dominant $O V$ and $V O$ blocks, respectively. This is also indicated from the NO analyses, wherein all NO pairs are made up of one orbital that is similar to an occupied orbital and the other is similar to an unoccupied orbital.

The above analyses of response 1PDMs given by Eq. (22) alone, however, does not allow us to construct an orbital picture of the SFG process as shown in Fig. 3. Next, we consider the response 1PDMs given by Eq. (24) corresponding to the dominant $\beta$ components. In this case, we analyze $\tilde{\gamma}_{\omega, \omega}^{x z, \pm}$, $\tilde{\gamma}_{\omega, \omega}^{x x, \pm}$, and $\tilde{\gamma}_{\omega, \omega}^{z y, \pm}$ response 1 PDMs corresponding to $\beta^{x x z}, \beta^{x z x}$, and $\beta^{z y y}$, respectively (see Table S4 in Supplementary Material). $\tilde{\gamma}_{\omega, \omega}^{x x, \pm}$ has the largest norms among all the components followed by $\tilde{\gamma}_{\omega, \omega}^{x y, \pm}$ (does not contribute to $\beta$ by symmetry) and $\tilde{\gamma}_{\omega, \omega}^{x z, \pm}$

The NO analysis of $\tilde{\gamma}_{\omega, \omega}^{x z,-}$ gives an NO pair characterized by a $b_{2} \rightarrow a_{2}$ orbital transition that is similar to the NTOs of the $\mathrm{XA}_{1} \rightarrow 1 \mathrm{~B}_{1}$ transition. This suggests that the $O V$ block of this response $1 \mathrm{PDM}$ is dominant, primarily because of the large transition moment between $\mathrm{XA}_{1}$ and $1 \mathrm{~B}_{1}$ states contributing to the signed sixth $\omega \mathrm{DM}$ in Eq. (24). The NO analysis of $\tilde{\gamma}_{\omega, \omega}^{x z,+}$ gives the same NO pair as the NO pair characterizing $\tilde{\gamma}_{\omega, \omega}^{x z,-}$ but with hole and particle NOs swapped. This suggests that $\tilde{\gamma}_{\omega, \omega}^{x z++}$ has the $V O$ block dominant, primarily due to the large transition moment between $\mathrm{XA}_{1}$ and $1 \mathrm{~B}_{1}$ states contributing to the signed fourth $\omega \mathrm{DM}$ in Eq. (24).

Next, we analyze the NOs of $\tilde{\gamma}_{\omega, \omega}^{x x,-}$ and $\tilde{\gamma}_{\omega, \omega}^{x x,+} \cdot \tilde{\gamma}_{\omega, \omega}^{x x,-}$ is characterized by two NO pairs. The NO pair with the dominant singular value gives a $b_{2} \rightarrow b_{2}$ orbital transition that is similar to the NTOs of the $\mathrm{XA}_{1} \rightarrow 2 \mathrm{~A}_{1}$ transition. This suggests a dominant $O V$ block for this response 1PDM. The second NO pair has the same $a_{2}$ orbital for the hole and particle NOs. This NO pair therefore originates from the $V V$ block to which the primary contribution comes from the third signed $\omega \mathrm{DM}$ in Eq. (24). The two NO pairs characterizing $\tilde{\gamma}_{\omega, \omega}^{x x,+}$ are the same as those of $\tilde{\gamma}_{\omega, \omega}^{x x,-}$ but with hole and particle NOs swapped. Further, $\theta=61^{\circ}$ between these signed response 1PDMs shows a significant deviation from $90^{\circ}$, which is consistent with the significance of the second NO pair arising from the $V V$ block.

$\tilde{\gamma}_{\omega, \omega}^{z y,-}$ with a dominant $O V$ block is characterized by the $b_{2} \rightarrow a_{1}$ NO transition. Here, $b_{2}$ NO resembles the hole NTO of the $\mathrm{XA}_{1} \rightarrow 1 \mathrm{~B}_{2}$ transition and $a_{1} \mathrm{NO}$ resembles a combination of the particle NTOs of the $\mathrm{XA}_{1} \rightarrow 1 \mathrm{~B}_{2}$ and $\mathrm{XA}_{1} \rightarrow 2 \mathrm{~B}_{2}$ transitions. $\tilde{\gamma}_{\omega, \omega}^{z,+}$ has a dominant $V O$ block and is characterized by the same NO pair but with swapped hole and particle NOs relative to the NO pair characterizing $\tilde{\gamma}_{\omega, \omega}^{y,-}$.

Similar to the NO analysis of response 1PDMs formalized according to Eq. (22), the NO analyses of response 1PDMs formalized according to Eq. (24) alone do not give the complete picture of SFG process. However, we now combine the analyses of $\gamma_{\varepsilon, \omega_{b}, \omega_{c}}^{b c,+}$ and $\tilde{\gamma}_{\varepsilon, \omega_{b}, \omega_{c}}^{a c,-}$ on the basis of Eq. (42) to characterize the two- and one-photon virtual states. For example, based on the orbital information from the NO analyses of $\gamma_{\omega, \omega}^{x z,+}$ and $\tilde{\gamma}_{\omega, \omega}^{x z,-}$ corresponding to $\beta^{x x z}$, the two- and one-photon virtual states are characterized as $b_{2} \stackrel{\omega_{x}+\omega_{z}}{\longrightarrow} a_{2}$ and $b_{2} \stackrel{\omega_{x}}{\longrightarrow} a_{2}$, respectively. This helps identify identify one important SFG orbital channel: $b_{2} \stackrel{\omega_{x}}{\longrightarrow} a_{2} \stackrel{\omega_{z}}{\longrightarrow} a_{2} \stackrel{-\omega_{x}^{\prime}}{\longrightarrow} b_{2}$ (Fig. 3(a)). For the $\beta^{x z x}$ component, the NO analyses of $\gamma_{\omega, \omega}^{z x,+}$ and $\tilde{\gamma}_{\omega, \omega}^{x x,-}$ identifies two SFG orbital channels: $b_{2} \stackrel{\omega_{z}}{\longrightarrow} \tilde{b}_{2} \stackrel{\omega_{x}}{\longrightarrow} a_{2} \stackrel{-\omega_{x}^{\prime}}{\longrightarrow} b_{2}$ (Fig. 3(b)) and $b_{2} \stackrel{\omega_{x}}{\longrightarrow} a_{2} \stackrel{\omega_{z}}{\longrightarrow} a_{2} \stackrel{-\omega_{x}^{\prime}}{\longrightarrow} b_{2}$ (Fig. 3(a)). Here, we use $\tilde{b}_{2}$ to distinguish the second $b_{2}$ orbital from the first $b_{2}$ orbital in the channel. For the $\beta^{z y y}$ component, the NO analyses of $\gamma_{\omega, \omega}^{y y,+}$ and $\tilde{\gamma}_{\omega, \omega}^{z,-}$ identifies one SFG orbital channel $b_{2} \stackrel{\omega_{y}}{\longrightarrow} a_{1} \stackrel{\omega_{y}}{\longrightarrow} \tilde{b}_{2} \stackrel{-\omega_{z}}{\longrightarrow} b_{2}$ as shown in Fig. 3(d).

\section{Damped one-photon resonant $\beta_{0}\left(-\omega_{1}-\omega_{2} ; \omega_{1}, \omega_{2} ; \varepsilon\right)$}

In this section, we analyze the orbitals involved in a SFG process wherein one of the absorbed photon energies is resonant with the excitation energy of an one-photon bright transition. Here, we pick $\omega_{b}$ that equals the excitation energy (8.15 $\mathrm{eV}$ ) of $1 \mathrm{~B}_{1}$ state, which dominates the UV-visible spectrum in the low-energy range. Based on our analysis in Fig. 2, the contribution of the $1 \mathrm{~B}_{1}$ state as an intermediate in the sum over states would thus be to the imaginary components of $\beta$, provided that the other energy-difference term in the denominator of the SOS expressions is greater than $\varepsilon$. However, for this calculation, $\omega_{c}$ equals $0.12 \mathrm{eV}$, which is smaller than $\varepsilon$ of $0.27 \mathrm{eV}$ (0.01 a.u.). This suggests that the SOS term with both intermediate states being $1 \mathrm{~B}_{1}$ instead contributes significantly to the real part of $\beta^{x x z}$. The real part of the $x x z$ component of $\beta$ is dominant, which is consistent with the two-state model involving the large dipole moment of the $1 \mathrm{~B}_{1}$ and its transition moment with the ground state and energy difference terms, $E_{1 \mathrm{~B}_{1}}-E_{\mathrm{XA}_{1}}-\omega_{b}$ and $E_{1 \mathrm{~B}_{1}}-E_{\mathrm{XA}_{1}}-\omega_{c}$, which are smaller than $\varepsilon$. The imaginary $\beta^{x x z}$ is also large and gets contribution from SOS terms with $E_{1 \mathrm{~B}_{1}}-E_{\mathrm{XA}_{1}}-\omega_{b}=0$ and the other energy-difference term greater than $\varepsilon$. The NO analyses of $\gamma_{\omega_{b}, \omega_{c}}^{x z,+, \operatorname{Re} / \mathrm{Im}}$ and $\tilde{\gamma}_{\omega_{b}, \omega_{c}}^{x z,-\operatorname{Re} / \mathrm{Im}}$ response 1PDMs corresponding the $\beta^{x x z}$ is given in Tables S5 and S6 of the Supplementary Material. Both the real and imaginary components have dominant $O V$ blocks, each characterized by a $O \rightarrow V$ NO transition. Using these NO transitions, the important orbital channel for this component is given by $b_{2} \stackrel{\omega_{x}}{\longrightarrow} a_{2} \stackrel{\omega_{z}}{\longrightarrow} a_{2} \stackrel{-\omega_{x}^{\prime}}{\longrightarrow} b_{2}$ (Fig. 3(a)), where $b_{2}$ and $a_{2}$ NOs resemble the NTOs corresponding to the $\mathrm{XA}_{1} \rightarrow 1 \mathrm{~B}_{1}$ transition.

$\beta^{z x x}$ is the next dominant component with its imaginary part larger in magnitude than its real part. This is consistent with the three-state model involving the $\mathrm{XA}_{1}, 2 \mathrm{~A}_{1}$, and $1 \mathrm{~B}_{1}$ states in which the dominant sum over states involves $E_{2 \mathrm{~A}_{1}}-E_{\mathrm{XA}_{1}}-$ $\omega_{a}>\varepsilon$ and $E_{1 \mathrm{~B}_{1}}-E_{\mathrm{XA}_{1}}-\omega_{b}=0$ along with the large dipole moment of the $1 \mathrm{~B}_{1}$ state and its large transition moment with the $\mathrm{XA}_{1}$ state. The corresponding $\mathrm{NO}$ analyses of $\gamma_{\omega_{b}, \omega_{c}}^{x x,+, \mathrm{Re} / \mathrm{Im}}$ and $\tilde{\gamma}_{\omega_{b}, \omega_{c}}^{x,-, R e / I m}$ response 1PDMs, with dominant $O V$ blocks, give a $b_{2} \stackrel{\omega_{x}}{\longrightarrow} a_{2} \stackrel{\omega_{x}}{\longrightarrow} \tilde{b}_{2} \stackrel{-\omega_{x}^{\prime}}{\longrightarrow} b_{2}$ (Fig. 3(c)), where the $b_{2}$ and $\tilde{b}_{2}$ NOs resemble the NTOs corresponding to the $\mathrm{XA}_{1} \rightarrow 2 \mathrm{~A}_{1}$ transition and the $a_{2}$ NO resembles the particle NTO of the $\mathrm{XA}_{1} \rightarrow 1 \mathrm{~B}_{1}$ transition. This also indicates that this orbital 
channel involving the one-photon resonant $b_{2} \stackrel{\omega_{x}}{\rightarrow} a_{2}$ transition is damped with a primary contribution to the imaginary part of $\beta^{z x x}$.

The third important component of the $\beta$ tensor is its $x z x$ component. With the same three-state model involving the $\mathrm{XA}_{1}, 2 \mathrm{~A}_{1}$, and $1 \mathrm{~B}_{1}$ states, we can explain that the real part is larger than the imaginary part as $E_{2 \mathrm{~A}_{1}}-E_{\mathrm{XA}_{1}}-\omega_{b} \in$ $\left\{\frac{\varepsilon(1+\chi)}{\chi-1}, \frac{\varepsilon(1-\chi)}{\chi+1}\right\}$, where $E_{2 \mathrm{~A}_{1}}-E_{\mathrm{XA}_{1}}+\omega_{a}=\chi \varepsilon$ as explained in Fig. 2(e). The NO analyses of $\gamma_{\omega_{b}, \omega_{c}}^{x,+, \operatorname{Re} / \mathrm{Im}}$ and $\tilde{\gamma}_{\omega_{b}, \omega_{c}}^{x x,-, \operatorname{Re} / \mathrm{Im}}$ response 1PDMs corresponding to $\beta^{x z x}$ suggests that the important orbital channel for both the real and imaginary components is the $b_{2} \stackrel{\omega_{z}}{\longrightarrow} \tilde{b}_{2} \stackrel{\omega_{x}}{\longrightarrow} a_{2} \stackrel{-\omega_{x}^{\prime}}{\longrightarrow} b_{2}$ channel (Fig. 3(b)).

\section{Damped two-photon resonant $\beta_{0}(-2 \omega ; \omega, \omega ; \varepsilon)$}

In this section, we characterize a two-photon resonant SFG transition with degenerate absorbed photons. Here, the sum frequency is resonant with the $\mathrm{XA}_{1} \rightarrow 2 \mathrm{~A}_{1}$ transition; so we expect the $2 \mathrm{~A}_{1}$ state to make significant contributions as an intermediate state in the sum over states to the imaginary $\beta$ components on the basis of Fig. 2. Consistent with this, the dominant contribution to the $\beta$ tensor comes from the imaginary zyy component, which is significantly influenced by $E_{2 \mathrm{~A}_{1}}-E_{\mathrm{XA}_{1}}+\omega_{a}=0$ and large transition moments between $\mathrm{B}_{2}$ states and $\mathrm{XA}_{1} / 2 \mathrm{~A}_{1}$ states, based on few-state models. The NO analyses (see Tables S7 and S8 of the Supplementary Material) of the $O V$-block-dominated $\gamma_{\omega_{b}, \omega_{c}}^{y y,+, \operatorname{Im}}$ and $\tilde{\gamma}_{\omega_{b}, \omega_{c}}^{z,,-\operatorname{Im}}$ response 1PDMs corresponding to $\beta^{z y y, \operatorname{Im}}$ suggests that the primary orbital channel involved is the $b_{2} \stackrel{\omega_{y}}{\longrightarrow} a_{1} \stackrel{\omega_{y}}{\longrightarrow} \tilde{b}_{2} \stackrel{-\omega_{z}}{\longrightarrow} b_{2}$ channel (Fig. 3(d)), where $b_{2}$ and $a_{1}$ NOs resemble the NTOs involved in the $\mathrm{XA}_{1} \rightarrow 1 \mathrm{~B}_{2}$ transition and $\tilde{b}_{2}$ NO resembles the particle NTO of the $\mathrm{XA}_{1} \rightarrow 2 \mathrm{~A}_{1}$ transition. This twophoton resonant channel is effectively damped and projected into the imaginary part of $\beta^{z y y}$ in the damped response calculation.

Another important two-photon resonant channel that is damped comes from the analysis of $\beta^{z x x}$, Im and its corresponding response 1PDMs. This component is again influenced by $E_{2 \mathrm{~A}_{1}}-E_{\mathrm{XA}_{1}}+\omega_{a}=0$ and large transition moments of the $1 \mathrm{~B}_{1}$ state with $\mathrm{XA}_{1}$ and $2 \mathrm{~A}_{1}$ states, based on few-state models. This is reflected in the NO analyses of the corresponding $\gamma_{\omega_{b}, \omega_{c}}^{x x,+\operatorname{Im}}$ and $\tilde{\gamma}_{\omega_{b}, \omega_{c}}^{x,-, \operatorname{Im}}$ response 1PDMs. Here, $\gamma_{\omega_{b}, \omega_{c}}^{x,+, \operatorname{Im}}$ is characterized by a single $b_{2} \rightarrow \tilde{b}_{2}$ NO transition (resembling the NTO pair in the $\left.\mathrm{XA}_{1} \rightarrow 2 \mathrm{~A}_{1}\right)$. In contrast, $\tilde{\gamma}_{\omega_{b}, \omega_{c}}^{x,-, \operatorname{Im}}$ is characterized by two NO transitions, $b_{2} \rightarrow a_{2}$ (resembling the NTO pair in the $\mathrm{XA}_{1} \rightarrow 1 \mathrm{~B}_{1}$ ) and $a_{2} \rightarrow \tilde{b}_{2}$ (resembling the NTO pair in the $1 \mathrm{~B}_{1} \rightarrow 2 \mathrm{~A}_{1}$ ), reflecting the impact of the large transition moments of $1 \mathrm{~B}_{1}$ state with $\mathrm{XA}_{1}$ and $2 \mathrm{~A}_{1}$ states. This NO analyses therefore identify a single dominant $b_{2} \stackrel{\omega_{x}}{\longrightarrow} a_{2} \stackrel{\omega_{x}}{\longrightarrow} \tilde{b}_{2} \stackrel{-\omega_{z}}{\longrightarrow} b_{2}$ orbital channel (Fig. 3(c)) for $\beta^{z x x, \operatorname{Im}}$.

In contrast, the important orbital channels that are not twophoton resonant are the $b_{2} \stackrel{\omega_{z}}{\longrightarrow} \tilde{b}_{2} \stackrel{\omega_{x}}{\longrightarrow} a_{2} \stackrel{-\omega_{x}^{\prime}}{\longrightarrow} b_{2}$ (Fig. 3(b)) and $b_{2} \stackrel{\omega_{x}}{\longrightarrow} a_{2} \stackrel{\omega_{z}}{\longrightarrow} a_{2} \stackrel{-\omega_{x}^{\prime}}{\longrightarrow} b_{2}$ (Fig. 3(a)) channels from the response 1PDMs corresponding to the equivalent $x z x$ and $x x z$ components of $\beta$ with larger real parts than imaginary.

\section{Damped one-photon + two-photon resonant}

$\beta_{0}\left(-\omega_{b}-\omega_{c} ; \omega_{b}, \omega_{c} ; \varepsilon\right)$

In this section, we characterize SFG transitions in which one of the absorbed photons equals the excitation energy of an excited state and the sum frequency equals the excitation of another excited state. We expect a few orbital channels to be enhanced via double resonance (one-photon plus twophoton resonance enhancement) and also orbital channels that are enhanced either by one-photon or two-photon resonance. The doubly resonance enhanced channels accumulate in the real components of the response 1PDMs along with the offresonance channels as explained in Fig. 2. Below, we consider two sets of absorbed photons for this double-resonance enhanced $\beta$ tensor.

First, we consider a double resonance enhancement SFG transition in which $\omega_{b}$ is resonant with the $\mathrm{XA}_{1} \rightarrow 1 \mathrm{~B}_{1}$ transition and the sum frequency is resonant with the $\mathrm{XA}_{1} \rightarrow 2 \mathrm{~A}_{1}$ transition. The dominant doubly resonance enhanced channel is characterized as $b_{2} \stackrel{\omega_{x}}{\longrightarrow} a_{2} \stackrel{\omega_{x}^{\prime}}{\longrightarrow} \tilde{b}_{2} \stackrel{-\omega_{z}}{\longrightarrow} b_{2}$ (Fig. 3(c)) and is obtained from the NO analyses of $\gamma_{\omega_{b}, \omega_{c}}^{x x,+, \operatorname{Re}}$ and $\tilde{\gamma}_{\omega_{b}, \omega_{c}}^{x,-, R e}$ response 1PDMs corresponding to $\beta^{z x x}$, Re (see Tables S9 and $\mathrm{S} 10$ of the Supplementary Material). Yet another orbital channel, $b_{2} \stackrel{\omega_{x}}{\longrightarrow} a_{2} \stackrel{\omega_{z}}{\longrightarrow} a_{2} \stackrel{-\omega_{x}^{\prime}}{\longrightarrow} b_{2}$ (Fig. 3(a)), which is enhanced by one-photon resonance, is obtained from the NO analyses of $\gamma_{\omega_{b}, \omega_{c}}^{x z,+ \text { Im }}$ and $\tilde{\gamma}_{\omega_{b}, \omega_{c}}^{x z,- \text { Im }}$ response 1PDMs corresponding to $\beta^{z x x, \operatorname{Im}}$. In contrast, the $b_{2} \stackrel{\omega_{z}}{\longrightarrow} \tilde{b}_{2} \stackrel{\omega_{x}}{\longrightarrow} a_{2} \stackrel{-\omega_{x}^{\prime}}{\longrightarrow} b_{2}$ orbital channel (Fig. 3(b)) obtained from NO analyses of response 1PDMs corresponding to $\beta^{x z x, \mathrm{Re}}$ is not enhanced by one-photon or two-photon resonance. Instead, this orbital channel is significant due to the large transition moments of the $1 \mathrm{~B}_{1}$ state with the $\mathrm{XA}_{1}$ and $2 \mathrm{~A}_{1}$ states. Here, the $b_{2}$ and $a_{2}$ NOs resemble the NTOs of the $\mathrm{XA}_{1} \rightarrow 1 \mathrm{~B}_{1}$ transition and $\tilde{b}_{2}$ NO resembles the particle $\mathrm{NTO}$ of the $\mathrm{XA}_{1} \rightarrow 2 \mathrm{~A}_{1}$ transition.

Next, we consider the SFG transition in which $\omega_{b}$ is resonant with the $\mathrm{XA}_{1} \rightarrow 1 \mathrm{~B}_{2}$ transition and the sum frequency is resonant with the $\mathrm{XA}_{1} \rightarrow 1 \mathrm{~A}_{2}$ transition. However, since the $\mathrm{XA}_{1} \rightarrow 1 \mathrm{~A}_{2}$ one-photon transition is dark, we do not expect to obtain orbital channels that are enhanced by double resonance. Consistent with this, the calculated $\beta$ tensor confirms this; $\beta^{a b c, I m}$ components are dominant and not $\beta^{a b c, \mathrm{Re}}$, which suggests that the dominant orbital channels are instead enhanced by one-photon (near-)resonance. The two orbital channels, $b_{2} \stackrel{\omega_{y}}{\longrightarrow} a_{1} \stackrel{\omega_{z}}{\longrightarrow} \tilde{a}_{1} \stackrel{-\omega_{y}^{\prime}}{\longrightarrow} b_{2}$ (Fig. 3(e)) and $b_{2} \stackrel{\omega_{y}}{\longrightarrow} a_{1} \stackrel{\omega_{y}}{\longrightarrow} \tilde{b}_{2} \stackrel{-\omega_{z}}{\longrightarrow} b_{2}$ (Fig. 3(d)), obtained from NO analyses of response 1PDMs corresponding to $\beta^{y y z, \operatorname{Im}}$ and $\beta^{z y y, \operatorname{Im}}$, respectively, feature the one-photon resonant $b_{2} \stackrel{\omega_{y}}{\longrightarrow} a_{1}$ orbital transition that characterizes the $\mathrm{XA}_{1} \rightarrow 1 \mathrm{~B}_{2}$ transition (see Tables S11 and S12 of the Supplementary Material). Here, the $b_{2}$ and $a_{1}$ NOs resemble the NTOs of the $\mathrm{XA}_{1} \rightarrow 1 \mathrm{~B}_{1}$ transition, $\tilde{b}_{2}$ NO resembles the particle NTO of the $\mathrm{XA}_{1} \rightarrow 2 \mathrm{~A}_{1}$ transition, and $\tilde{a}_{1} \mathrm{NO}$ resembles a combination of the parti- 
cle NTOs of $\mathrm{XA}_{1} \rightarrow 1 \mathrm{~B}_{1}$ and $\mathrm{XA}_{1} \rightarrow 2 \mathrm{~B}_{1}$ transitions. Two other orbital channels, $b_{2} \stackrel{\omega_{x}}{\longrightarrow} a_{2} \stackrel{\omega_{z}}{\longrightarrow} a_{2} \stackrel{-\omega_{x}^{\prime}}{\longrightarrow} b_{2}$ (Fig. 3(a)) and $b_{2} \stackrel{\omega_{z}}{\longrightarrow} \tilde{b}_{2} \stackrel{\omega_{x}}{\longrightarrow} a_{2} \stackrel{-\omega_{x}^{\prime}}{\longrightarrow} b_{2}$ (Fig. 3(b)), are obtained from the response 1 PDMs corresponding to $\beta^{x x z, I m}$ and $\beta^{x z x, I m}$, respectively, due to the near-resonance of the sum frequency to the excitation energy of $1 \mathrm{~B}_{1}$ state, i.e., $E_{1 \mathrm{~B}_{1}}-E_{\mathrm{XA}_{1}}+\omega_{a}<\varepsilon$. Here, the $b_{2}$ and $a_{2}$ NOs resemble the NTOs of the $\mathrm{XA}_{1} \rightarrow$ $1 \mathrm{~B}_{1}$ transition and the $\tilde{b}_{2} \mathrm{NO}$ resembles the particle NTO of the $\mathrm{XA}_{1} \rightarrow 2 \mathrm{~A}_{1}$ transition.

\section{Undamped $\beta_{0}(0 ; 0,0 ; 0)$}

In this section, we characterize the response 1PDMs for the static case for which response 1PDMs from Eqs. (22) and (24) are the same.. In addition, $\gamma_{0,0}^{+}$and $\gamma_{0,0}^{-}$are the same. In addition, $\beta^{a b b}=\beta^{b a b}=\beta^{b b a}$; so only $\beta^{z x x}, \beta^{z y y}$, and $\beta^{z z z}$ are unique. Table S13 in the Supplementary Material gives the NOs for the different response 1PDMs. Based on $\Upsilon^{b c}$ values, the two most significant components are $y y$ and $x x$. For the $\gamma_{0,0}^{y y, R e}$ response 1PDM, there are two dominant pairs of NOs, each with the same NO for the hole and particle orbitals. This suggests that the dominant $\omega \mathrm{DMs}$ contribute to the $O O$ and $V V$ blocks of $\gamma_{0,0}^{y y, R e}$. Both NO pairs are made up of $b_{2}$ orbitals; the $b_{2} \mathrm{NO}$ in the first NO pair has a more complex nodal structure compared to the $b_{2}$ orbital (similar to the HOMO) in the second NO pair. The response 1PDM that is complementary to $\gamma_{0,0}^{y, \operatorname{Re}}$ for characterizing the $\beta^{z y y}$ component is $\gamma_{0,0}^{z y, \operatorname{Re}}$ for which the NO analysis features a $b_{2} \rightarrow a_{1}$ orbital transition and its reverse transition. Combining these NO analyses, $\beta^{z y y}$ features a $b_{2} \rightarrow a_{2} \rightarrow \tilde{b}_{2} \rightarrow b_{2}$.

For characterizing the $\beta^{z x x}$ component, we perform NO analyses of $\gamma_{0,0}^{x x, \operatorname{Re}}$ and $\gamma_{0,0}^{z x, \mathrm{Re}}$. The dominant NO pair for $\gamma_{0,0}^{x x, R e}$ comprises a $b_{2}$ orbital for both the hole and particle orbitals. The second significant NO pair comprises a $a_{2}$ orbital for both the hole and particle orbitals. This suggests that the dominant $\omega \mathrm{DMs}$ contribute to the $V V$ and $O O$ blocks of $\gamma_{0,0}^{x x, \operatorname{Re}}$. The NO analysis of $\gamma_{0,0}^{z x, R e}$ features two NO pairs; the first gives a $b_{2} \rightarrow a_{2}$ transition and the second is the reverse of the first. Combining these NO analyses, $\beta^{z x x}$ features a $b_{2} \rightarrow a_{2} \rightarrow b_{2} \rightarrow b_{2}$ and $b_{2} \rightarrow a_{2} \rightarrow a_{2} \rightarrow b_{2}$ channels.

The $\beta^{z z z}$ component can be characterized by the NO analysis of $\gamma_{0,0}^{z z, R e}$, which features two NO pairs of opposing direction; each made up of the same pair of $a_{1}$ orbitals. So, $\beta^{z z z}$ is characterized by $a_{1} \rightarrow \tilde{a}_{1} \rightarrow \tilde{a}_{1} \rightarrow a_{1}$ and $a_{1} \rightarrow a_{1} \rightarrow \tilde{a}_{1} \rightarrow a_{1}$ channels.

\section{B. para-Nitroaniline}

Table III gives the excitation energies and permanent dipole moments of the low-lying excited $\mathrm{A}_{1}$ and $\mathrm{B}_{1}$ states of paranitroaniline. It also provides the oscillator strengths and NTOs for the transition to these states from the ground state. The oscillator strengths for the $\mathrm{XA}_{1} \rightarrow 4 \mathrm{~A}_{1}, \mathrm{XA}_{1} \rightarrow 2 \mathrm{~A}_{1}$, and $\mathrm{XA}_{1} \rightarrow 3 \mathrm{~B}_{1}$ transitions are the largest. The $\mathrm{XA}_{1} \rightarrow 2 \mathrm{~A}_{1}$ is characterized as a transition between a hole $b_{2}$ (resembling HOMO) and a particle $b_{2}$ NTOs with a large $z_{h \rightarrow e}$. The permanent dipole moment of the $2 \mathrm{~A}_{1}$ state is larger than the dipole moment of the ground state. These quantities and NTOs suggest a large intra-molecular charge transfer from the amine end to the nitro end of the molecule. In contrast, the $\mathrm{XA}_{1} \rightarrow 4 \mathrm{~A}_{1}$ transition is characterized using two NTO pairs, $a_{2} \rightarrow a_{2}$ and $b_{2} \rightarrow b_{2}$ with a small $z_{h \rightarrow e}$. The $4 \mathrm{~A}_{1}$ state has a permanent dipole moment that is slightly smaller than that of the ground state. This suggest that the intra-molecular charge-transfer character of the $\mathrm{XA}_{1} \rightarrow 4 \mathrm{~A}_{1}$ transition is negligible. The $\mathrm{XA}_{1} \rightarrow 3 \mathrm{~B}_{1}$ transition is characterized by a $a_{2} \rightarrow b_{2}$ NTO transition with a small $z_{h \rightarrow e}$, indicating negligible intra-molecular charge-transfer character. Below, we discuss the wave-function analysis of damped response 1PDMs for three SFG processes, which are two-photon resonant with the $\mathrm{XA}_{1} \rightarrow 2 \mathrm{~A}_{1}, \mathrm{XA}_{1} \rightarrow 4 \mathrm{~A}_{1}$, and $\mathrm{XA}_{1} \rightarrow 3 \mathrm{~B}_{1}$ transitions with degenerate photons. We also perform this analysis for the undamped static case. In all these cases, the $\beta^{z z z}$ component of the first hyperpolarizability tensor dominates (Table S14 of the Supplementary Material). So, the focus below is primarily on the $\gamma_{\omega_{1}, \omega_{2}}^{z,+, R e / I m}$ and $\tilde{\gamma}_{\omega_{1}, \omega_{2}}^{z,-, R e / I m}$ response 1PDMs. The $\mathrm{NO}$ analysis of these response 1PDMs is given in Tables S15 and S16 of the Supplementary Material. The important orbital channels across these different cases are shown in Fig. 4. Quantities such as $d_{\mathrm{exc}}^{ \pm}, d_{h \rightarrow e}^{ \pm}$, and $\mathcal{Q}^{ \pm}$associated with all the component response 1PDMs and the overall SFG processes are given in Tables S17 and S18 of the Supplementary Material. Here, $\mathcal{Q}^{ \pm}$is computed between the amine half (fragment $A$ ) and nitro half (fragment $B$ ) of the molecule.

\section{Undamped $\beta_{0}(-2 \omega ; \omega, \omega ; 0)$}

For the undamped static case, $\gamma_{0,0}^{z z,+, \mathrm{Re} / \mathrm{Im}}$ and $\tilde{\gamma}_{0,0}^{z,-, \mathrm{Re} / \mathrm{Im}}$ are equivalent. The NO analysis of these response 1PDMs is dominated by two NO pairs. The hole and particle orbitals in each of these NO pairs look similar and have $b_{2}$ symmetry, indicating that the $O O$ and $V V$ blocks of the response 1PDMs are dominant. The $b_{2}$ orbitals involved in both the NO pairs are given by linear combinations of the hole and particle orbitals of the $\mathrm{XA}_{1} \rightarrow 2 \mathrm{~A}_{1}$ transition. This suggests that the character of the important virtual state is dominated by the $\mathrm{XA}_{1} \rightarrow 2 \mathrm{~A}_{1}$ transition. The $\omega \mathrm{DMs}$ that contribute to the $O O$ and $V V$ blocks of the response 1PDMs are dominant due to the large change in dipole moment between the $2 \mathrm{~A}_{1}$ and $\mathrm{XA}_{1}$ states, which is also larger than the transition dipole moment of this transition. Despite the dominant contribution of this intra-molecular charge-transfer transition to the static $\beta^{z z z}$, the corresponding $d_{h \rightarrow e}^{ \pm}$is negligible (0.06 $\AA$ ), a consequence of the similar hole and particle orbitals in the dominant NO pairs.

\section{Damped two-photon resonant $\beta_{0}(-2 \omega ; \omega, \omega ; \varepsilon)$}

Here, we first consider the SFG process wherein two degenerate photons are absorbed and the sum-frequency photon is 
TABLE III. Excitation energies $(\Omega, \mathrm{eV})$, oscillator strengths $(f)$, permanent dipole moments ${ }^{a}(\langle\mu\rangle$, a.u.), and wave-function analysis for lowlying $\mathrm{A}_{1}$ and $\mathrm{B}_{1}$ excited states in para-nitroaniline. The wave-function analysis for each transition includes the norm of transition 1PDM $(\|\gamma\|)$, the size of the exciton $\left(d_{\mathrm{exc}}\right)$, the vectorial distance between the centroids of the hole and electron distributions $\left(d_{h \rightarrow e}\right)$, participation ratio $(P R)$, and dominant NTO pairs (isovalue is 0.01$)$. The singular values $\left(\sigma_{K}\right)$ correspond to normalized transition 1 PDMs. EOM-EECCSD/aug-cc-pVDZ.

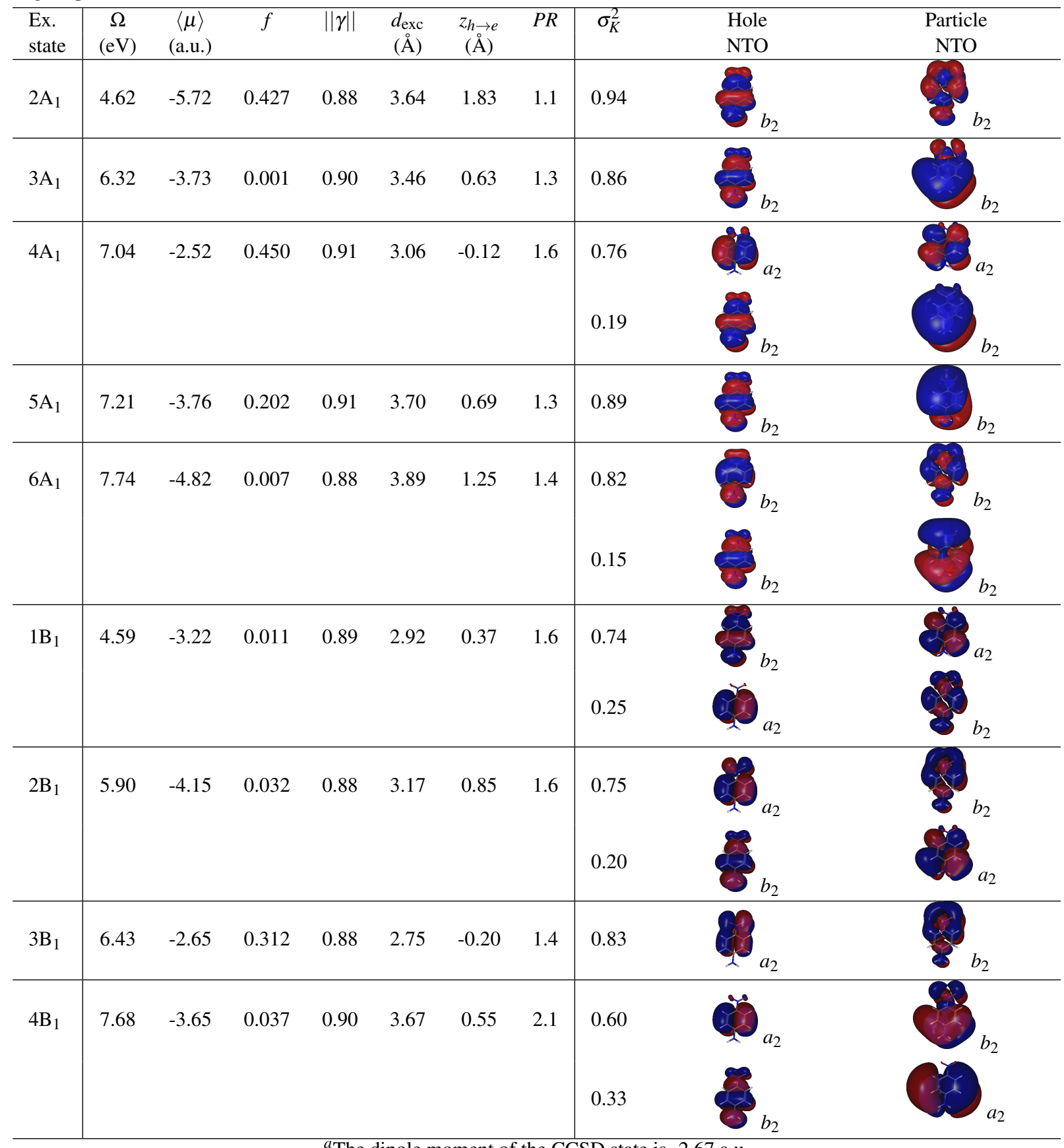

${ }^{a}$ The dipole moment of the CCSD state is -2.67 a.u.

resonant with the excitation energy of the $2 \mathrm{~A}_{1}$ state. Considering the large oscillator strength for the $\mathrm{XA}_{1} \rightarrow 2 \mathrm{~A}_{1}$ transition and the large change in permanent dipole moment between these two states, we expect this transition to dominate $\beta^{z z z}$. The choice of the sum-frequency photon then ensures that the imaginary component of $\beta^{z z z}$ is larger than the real component, since no other $A_{1}$ state lies in the vicinity of the $2 A_{1}$ state with an excitation energy of $\Omega_{2 \mathrm{~A}_{1}}$ such that $\Omega_{2 \mathrm{~A}_{1}}-\omega_{1 / 2} \in$ $\{-\varepsilon, \varepsilon\}$ for epsilon $=0.05$ a.u. $(1.36 \mathrm{eV})$. Consistent with this, the damped resonance-enhanced contribution primarily enters $\beta^{z z z, I m}$, which is approximately three times $\beta^{z z z, \operatorname{Re}}$. The NO analysis of the corresponding $\gamma_{\omega, \omega}^{z z,+, \operatorname{Im}}$ and $\tilde{\gamma}_{\omega, \omega}^{z,-,}$,Im gives similar NO pair that is characterized as a $b_{2} \rightarrow \tilde{b}_{2}$ transition with the hole and particle NOs similar to the NTOs for the 


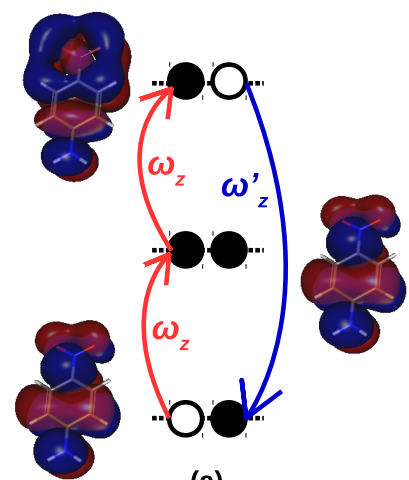

(a)

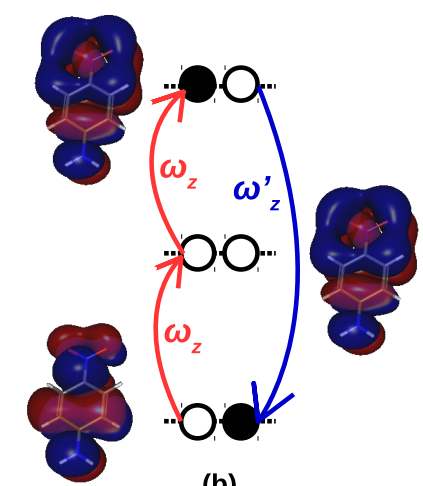

(b)

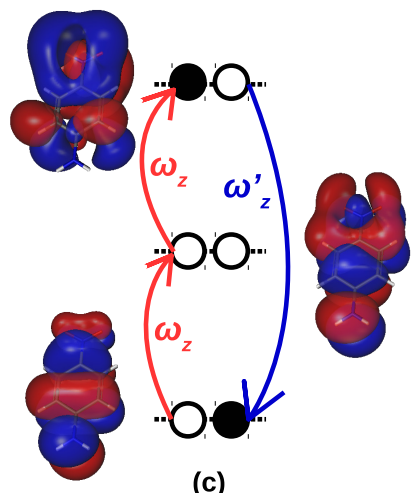

(c)

FIG. 4. The important orbital channels for para-nitroaniline in the SFG process for different sets of photon energies obtained from the NO analyses of corresponding response 1PDMs. (a) The $b_{2} \stackrel{\omega_{z}}{\longrightarrow} b_{2} \stackrel{\omega_{z}}{\longrightarrow} \tilde{b}_{2} \stackrel{-\omega_{z}^{\prime}}{\longrightarrow} b_{2}$ orbital channel. (b) The $b_{2} \stackrel{\omega_{z}}{\longrightarrow} \tilde{b}_{2} \stackrel{\omega_{z}}{\longrightarrow} \tilde{b}_{2} \stackrel{-\omega_{z}^{\prime}}{\longrightarrow} b_{2}$ orbital channel. (c) The $b_{2} \stackrel{\omega_{z}}{\longrightarrow} \tilde{b}_{2} \stackrel{\omega_{z}}{\longrightarrow} \tilde{\tilde{b}}_{2} \stackrel{-\omega_{z}^{\prime}}{\longrightarrow} b_{2}$ orbital channel. Here, $b_{2}$ and $\tilde{b}_{2}$ NOs resemble the hole and particle NTOs of the XA $\rightarrow 2 \mathrm{~A}_{1}$ transition. $\tilde{\tilde{b}}_{2} \mathrm{NO}$ is similar to the $\tilde{b}_{2} \mathrm{NO}$, indicating tht the contribution from other $\mathrm{XA}_{1} \rightarrow \mathrm{nA}_{1}$ transitions is non-negligible in addition to the dominant contribution from the $\mathrm{XA}_{1} \rightarrow 2 \mathrm{~A}_{1}$ transition. Here, $\omega_{z}$ is the energy of absorbed photons and $\omega_{z}^{\prime}$ is the energy of the sum-frequency photon.

$\mathrm{XA}_{1} \rightarrow 2 \mathrm{~A}_{1}$ transition. From these NO analyses, we identify two dominant orbital channels: $b_{2} \stackrel{\omega_{z}}{\longrightarrow} \tilde{b}_{2} \stackrel{\omega_{z}}{\longrightarrow} \tilde{b}_{2} \stackrel{-2 \omega_{z}}{\longrightarrow} b_{2}$ (Fig. 4(a)) and $b_{2} \stackrel{\omega_{z}}{\longrightarrow} b_{2} \stackrel{\omega_{z}}{\longrightarrow} \tilde{b}_{2} \stackrel{-2 \omega_{z}}{\longrightarrow} b_{2}$ (Fig. 4(b)).

Next, we consider the SFG process wherein two degenerate photons are absorbed and the sum-frequency photon is resonant with the excitation energy of the $3 \mathrm{~B}_{1}$ state. Although the transition dipole moment for the $\mathrm{XA}_{1} \rightarrow 3 \mathrm{~B}_{1}$ transition is large, the negligible change in the permanent dipole moment between these states results in $\beta^{x x z}$ and $\beta^{x z x}$ not being dominant despite the resonant sum-frequency photon. Instead, the non-resonant contributions, primarily from the $\mathrm{XA}_{1} \rightarrow$ $2 \mathrm{~A}_{1}$ transition, to the $\beta^{z z z, \mathrm{Re}}$ dominate the first hyperpolarizability tensor as can be seen from the NO pair characterizing $\tilde{\gamma}_{\omega, \omega}^{z z,-, R e}$ that is similar to the $b_{2} \rightarrow \tilde{b}_{2}$ NTO pair for the $\mathrm{XA}_{1} \rightarrow 2 \mathrm{~A}_{1}$ transition. $\gamma_{\omega, \omega}^{z z,+, R e}$ is characterized by a $b_{2} \rightarrow \tilde{\tilde{b}}_{2}$ NO transition with the particle NO slightly different from the $\tilde{b}_{2}$, indicating that other $\mathrm{XA}_{1} \rightarrow \mathrm{nA}_{1}$ transitions also contribute to this response 1PDM. This suggests a dominant $b_{2} \stackrel{\omega_{z}}{\longrightarrow} \tilde{b}_{2} \stackrel{\omega_{z}}{\longrightarrow} \tilde{b}_{2} \stackrel{-2 \omega_{z}}{\longrightarrow} b_{2}$ orbital channel for this SFG process (Fig. 4(c)). We also note that one of the NO pair characterizing the $\gamma_{\omega, \omega}^{z z,+, \mathrm{Im}}$ response $1 \mathrm{PDM}$ is somewhat similar to the NTO pair for the $\mathrm{XA}_{1} \rightarrow 3 \mathrm{~A}_{1}$ transition due to the nearresonance of the sum-frequency photon with the excitation energy of the $3 \mathrm{~A}_{1}$ state. However, unlike the large $\Upsilon_{\omega, \omega}^{z z,+, \mathrm{Im}}$, $\tilde{\Upsilon}_{\omega, \omega}^{z z,-, I m}\left(\Upsilon\right.$ corresponding to $\left.\tilde{\gamma}_{\omega, \omega}^{z z,-, \operatorname{Im}}\right)$ is negligible and so the channel involving this NO pair is not dominant.

A similar $b_{2} \stackrel{\omega_{z}}{\longrightarrow} \tilde{b}_{2} \stackrel{\omega_{z}}{\longrightarrow} \tilde{\tilde{b}}_{2} \stackrel{-2 \omega_{z}}{\longrightarrow} b_{2}$ orbital channel also characterizes the dominant $\beta^{z z z, \text { Re }}$ associated with the SFG process with degenerate photons and sum-frequency photon resonant with the transition to the $4 \mathrm{~A}_{1}$ state. In this case, the energy of the absorbed photons is in the vicinity of the excitation energy of the $2 \mathrm{~A}_{1}$ state, which results in the damped contribution to enter primarily into the real component. As a result, the NO pair characterizing $\tilde{\gamma}_{\omega, \omega}^{z z,-, R e}$ is similar to the NTO pair of the $\mathrm{XA}_{1} \rightarrow 2 \mathrm{~A}_{1}$ transition. We also note that the corresponding imaginary response 1 PDMs give non-negligible contributions and are characterized by different $b_{2}$ NOs, indicating that a number of $A_{1}$ states contribute to these response 1PDMs.

We also compute $d_{h \rightarrow e}^{+} / \tilde{d}_{h \rightarrow e}^{-}$and $\mathcal{Q}^{+} / \tilde{\mathcal{Q}}^{-}$, which highlight the intra-molecular charge-transfer character of these three SFG processes. These quantities are largest for the SFG process for which the sum-frequency photon is resonant with the $2 \mathrm{~A}_{1}$ state. This larger separation of the centroids of the hole and electron distributions and the larger charge transferred from the amine half to the nitro half of the molecule is consistent with the larger magnitude of the $\beta^{z z z}$ compared to those for the other two SFG processes. This is also consistent with the simple Huckel model for a charge-transfer $\pi-\pi^{*}$ transition in which the transition dipole moment increases with increasing separation of the hole and electron orbitals. For the SFG processes that are two-photon resonant with the $\mathrm{XA}_{1} \rightarrow 4 \mathrm{~A}_{1}$ and $\mathrm{XA}_{1} \rightarrow 3 \mathrm{~B}_{1}$ transitions, the electronic charge transfer for the transitions to the two-photon virtual state is towards the nitro group as indicated by the (similar) positive $d_{h \rightarrow e}^{+}$. In contrast, the charge transfer for the transition to the one-photon virtual state is away from the nitro group as indicated by the negative $\tilde{d}_{h \rightarrow e}^{-}$. Whereas $\mathcal{Q}^{+}$and $\tilde{\mathcal{Q}}^{-}$for these SFG processes are similar, the more negative $\tilde{d}_{h \rightarrow e}^{-}$for the former SFG process suggests a smaller net charge transfer towards the nitro group and is consistent with a slightly smaller $\beta^{z z z}$ than that of the latter SFG process. For the SFG process that is twophoton resonant with the $\mathrm{XA}_{1} \rightarrow 2 \mathrm{~A}_{1}$ transition, both $d_{h \rightarrow e}^{+}$ and $\tilde{d}_{h \rightarrow e}^{-}$are positive.

\section{Hemibonded $\mathrm{OH}+\mathrm{H}_{2} \mathrm{O}$ complex}

In this section, we illustrate how the orbital picture for the SFG process in a system comprising an open-shell hydroxyl radical and a water molecule is constructed using response 
TABLE IV. Excitation energies $(\Omega, \mathrm{eV})$, oscillator strengths $(f)$, permanent dipole moments ${ }^{a}(\langle\mu\rangle$, a.u.), and wave-function analysis for lowlying excited states in the hemibonded $\mathrm{OH}+\mathrm{H}_{2} \mathrm{O}$ complex. The wave-function analysis for each transition includes the norm of transition 1PDM $(\| \gamma||)$, the size of the exciton $\left(d_{\mathrm{exc}}\right)$, the vectorial distance between the centroids of the hole and electron distributions $\left(d_{h \rightarrow e}\right)$, and dominant NTO pairs (isovalue is 0.01$)$. The singular values $\left(\sigma_{K}\right)$ correspond to normalized transition 1PDMs. UHF/EOM-EE-CCSD/aug-ccpVDZ,

\begin{tabular}{|c|c|c|c|c|c|c|c|c|c|c|c|c|}
\hline $\begin{array}{c}\text { Ex. } \\
\text { state }\end{array}$ & $\begin{array}{c}\Omega \\
(\mathrm{eV})\end{array}$ & $\begin{array}{c}\langle\mu\rangle \\
\text { (a.u.) }\end{array}$ & $f$ & $\|\gamma\|$ & $\begin{array}{l}d_{\mathrm{exc}} \\
(\AA)\end{array}$ & $\begin{array}{c}x_{h \rightarrow e} \\
(\AA)\end{array}$ & $\begin{array}{c}y_{h \rightarrow e} \\
(\AA)\end{array}$ & $\begin{array}{c}z_{h \rightarrow e} \\
(\AA)\end{array}$ & $\begin{array}{c}d_{h \rightarrow e} \\
(\AA)\end{array}$ & $\sigma_{K}^{2}$ & $\begin{array}{l}\text { Hole } \\
\text { NTO }\end{array}$ & $\begin{array}{c}\text { Particle } \\
\text { NTO }\end{array}$ \\
\hline $2 \mathrm{~A}$ & 0.19 & 0.33 & 0.000 & 0.97 & 1.19 & -0.01 & 0.00 & 0.00 & 0.01 & 1.00 & & \\
\hline $3 \mathrm{~A}$ & 4.66 & 0.44 & 0.009 & 0.97 & 1.20 & 0.01 & 0.12 & -0.09 & 0.15 & 0.99 & & \\
\hline $4 \mathrm{~A}$ & 6.45 & 4.21 & 0.053 & 0.92 & 2.87 & 2.21 & -0.09 & 0.15 & 2.21 & 0.99 & & \\
\hline
\end{tabular}

${ }^{a}$ The dipole moment of the CCSD state is 0.34 a.u.

1PDMs. Here, we consider a hemibonded complex between the radical and water, which is considered to give strong intermolecular charge-transfer peaks in the UV-visible spectrum of the aqueous solution of hydroxyl radical. The low-lying electronic transitions and their NTO analysis for this system are provided in Table IV, which shows a charge-transfer XA $\rightarrow 4 \mathrm{~A}$ transition at $6.45 \mathrm{eV}$ with a larger oscillator strength compared to a localized XA $\rightarrow 3 \mathrm{~A}$ transition at $4.66 \mathrm{eV}$ on the radical. This intermolecular charge transfer involves a $p_{x}\left(\mathrm{H}_{2} \mathrm{O}\right)$ hole NTO and a $p_{x}(\mathrm{OH} \cdot)$ particle NTO representing the lone pair on water and the half-filled $1 \pi\left(p_{x}\right)$ orbital on the radical. The localized XA $\rightarrow 3 \mathrm{~A}$ transition on the radical is characterized by a $\sigma$ hole NTO and the half-filled $p_{x}$ particle NTO. Below, we discuss the important components of the first hyperpolarizbility tensor (Table S19 of the Supplementary Material) and the wave-function analysis of damped response 1PDMs for two one-photon-resonant SFG processes in which one of the absorbed photons is resonant with either the $\mathrm{XA} \rightarrow 3 \mathrm{~A}$ transition or the $\mathrm{XA} \rightarrow 4 \mathrm{~A}$ transition. We compute and discuss the orbitals involved in these SFG processes as well as the associated quantities such as $d_{\mathrm{exc}}^{ \pm}, d_{h \rightarrow e}^{ \pm}$, and $\mathcal{Q}^{ \pm}$ (Tables S20-S25 of the Supplementary Material). Here, $\mathcal{Q}^{ \pm}$ is computed between the radical (fragment $A$ ) and the water molecule (fragment $B$ ).

For both the one-photon-resonant SFG processes, the real and imaginary $\beta^{x x x}$ components are dominant compared to the other components. For the SFG process that is one-photon resonant with the $\mathrm{XA} \rightarrow 3 \mathrm{~A}$ transition, these components are almost an order of magnitude smaller than those for the SFG process that is one-photon resonant with the XA $\rightarrow 4 \mathrm{~A}$ transition. This is expected as the latter involves resonance enhancement of the $\mathrm{XA} \rightarrow 4 \mathrm{~A}$ transition that has a much larger transition dipole moment compared to that of the $\mathrm{XA} \rightarrow 3 \mathrm{~A}$ transition. Here, we compute the NOs associated with the $\gamma_{\omega_{1}, \omega_{2}}^{x x,+, \operatorname{Re} / \mathrm{Im}}$ and $\tilde{\gamma}_{\omega_{1}, \omega_{2}}^{x x,-, \operatorname{Re} / \mathrm{Im}}$ response 1PDMs.

For the SFG process that is one-photon resonant with the $\mathrm{XA} \rightarrow 3 \mathrm{~A}$ transition, $\tilde{\gamma}_{\omega_{1}, \omega_{2}}^{x x,-, \operatorname{Re}}$ and $\tilde{\gamma}_{\omega_{1}, \omega_{2}}^{x x,- \text { Im }}$ response 1PDMs give different NO pairs. $\tilde{\gamma}_{\omega_{1}, \omega_{2}}^{x x, \text {,Im }}$ collects the damped contribution from the resonance enhancement of the $\mathrm{XA} \rightarrow 3 \mathrm{~A}$ transition such that the dominant NO pair involves a hole NO that is a combination of $\sigma\left(\mathrm{OH}^{\cdot}\right)$ and $p_{x}\left(\mathrm{H}_{2} \mathrm{O}\right)$ orbitals and a particle NO that resembles the $p_{x}\left(\mathrm{OH}^{-}\right)$orbital. The offresonance contributions are collected in $\tilde{\gamma}_{\omega_{1}}^{x x, \omega_{2}}$ for which the $p_{x}\left(\mathrm{H}_{2} \mathrm{O}\right) \rightarrow p_{x}\left(\mathrm{OH}^{\cdot}\right)$ NO pair suggests that the dominant contribution comes from the intermolecular charge-transfer XA $\rightarrow 4 \mathrm{~A}$ transition. On the other hand, $\gamma_{\omega_{1}, \omega_{2}}^{x x,+, \operatorname{Re}}$ and $\gamma_{\omega_{1}, \omega_{2}}^{x x,+\operatorname{Im}}$ response 1PDMs are characterized similarly by the dominant $p_{x}\left(\mathrm{H}_{2} \mathrm{O}\right) \rightarrow p_{x}\left(\mathrm{OH}^{*}\right)$ NO pair. From this $\mathrm{NO}$ analyses, the important SFG orbital channels involved are $p_{x}\left(\mathrm{H}_{2} \mathrm{O}\right)$ $\stackrel{\omega_{x}^{\prime}}{\longrightarrow} \sigma\left(\mathrm{OH}^{\cdot}\right)+p_{x}\left(\mathrm{H}_{2} \mathrm{O}\right) \stackrel{\omega_{x}}{\longrightarrow} p_{x}\left(\mathrm{OH}^{\cdot}\right) \stackrel{-\omega_{x}^{\prime \prime}}{\longrightarrow} p_{x}\left(\mathrm{H}_{2} \mathrm{O}\right), p_{x}\left(\mathrm{H}_{2} \mathrm{O}\right)$ $\stackrel{\omega_{x}^{\prime}}{\longrightarrow} p_{x}\left(\mathrm{H}_{2} \mathrm{O}\right) \stackrel{\omega_{x}}{\longrightarrow} p_{x}\left(\mathrm{OH} \cdot \stackrel{-\omega_{x}^{\prime \prime}}{\longrightarrow} p_{x}\left(\mathrm{H}_{2} \mathrm{O}\right)\right.$, and $p_{x}\left(\mathrm{H}_{2} \mathrm{O}\right) \stackrel{\omega_{x}^{\prime}}{\longrightarrow}$ $p_{x}\left(\mathrm{OH}^{\cdot}\right) \stackrel{\omega_{x}}{\longrightarrow} p_{x}\left(\mathrm{OH} \cdot \stackrel{-\omega_{x}^{\prime \prime}}{\longrightarrow} p_{x}\left(\mathrm{H}_{2} \mathrm{O}\right)\right.$ as shown in Fig. 5(a), $5(\mathrm{~b})$, and 5(c), respectively, where $\omega_{x}^{\prime \prime}$ is the energy of the sum-frequency photon.

For the SFG process that is one-photon resonant with the $\mathrm{XA} \rightarrow 4 \mathrm{~A}$ transition, the contribution from the resonantly enhanced XA $\rightarrow 4 \mathrm{~A}$ transition is collected primarily in $\tilde{\gamma}_{\omega_{1}, \omega_{2}}^{x x,-\operatorname{Im}}$ as confirmed by its characteristic $p_{x}\left(\mathrm{H}_{2} \mathrm{O}\right) \rightarrow p_{x}\left(\mathrm{OH}^{\cdot}\right)$ NO pair, although $\tilde{\gamma}_{\omega_{1}, \omega_{2}}^{x x,-, R e}$ is also characterized by the same NO pair. This NO pair also dominates the NO analyses of $\gamma_{\omega_{1}, \omega_{2}}^{x x,+R e}$ and $\gamma_{\omega_{1}, \omega_{2}}^{x x,+ \text { Im }}$ response 1PDMs, suggesting that the SFG process is characterized by channels (b) and (c) shown in Fig. 5.

The computed $d_{\mathrm{exc}}^{ \pm}, d_{h \rightarrow e}^{ \pm}$, and $\mathcal{Q}^{ \pm}$for these two SFG processes further confirms that the SFG process that is onephoton resonant with the $\mathrm{XA} \rightarrow 4 \mathrm{~A}$ transition is more strongly influenced by the intermolecular charge transfer from the lone pair on water to the half-filled orbital on the radical than the SFG process that is one-photon resonant with the $\mathrm{XA} \rightarrow 3 \mathrm{~A}$ transition. The former SFG process shows larger values for $\tilde{d}_{h \rightarrow e}^{-}$and $\tilde{\mathcal{Q}}^{-}$suggesting that the one-photon virtual state involves a larger charge transfer from water to the radical than in the latter SFG process. In particular, the dominant $\tilde{x}_{h \rightarrow e}^{-}$ component of $\tilde{d}_{h \rightarrow e}^{-}$is much smaller for the latter SFG process than that of the former, due to the strong influence of the localized XA $\rightarrow 3 \mathrm{~A}$ transition. The $\tilde{d}_{\mathrm{exc}}^{-}$is also larger for the former SFG process suggesting it is more delocalized than the latter SFG process. In contrast, the $d_{h \rightarrow e}^{+}$and $\mathcal{Q}^{+}$values are 

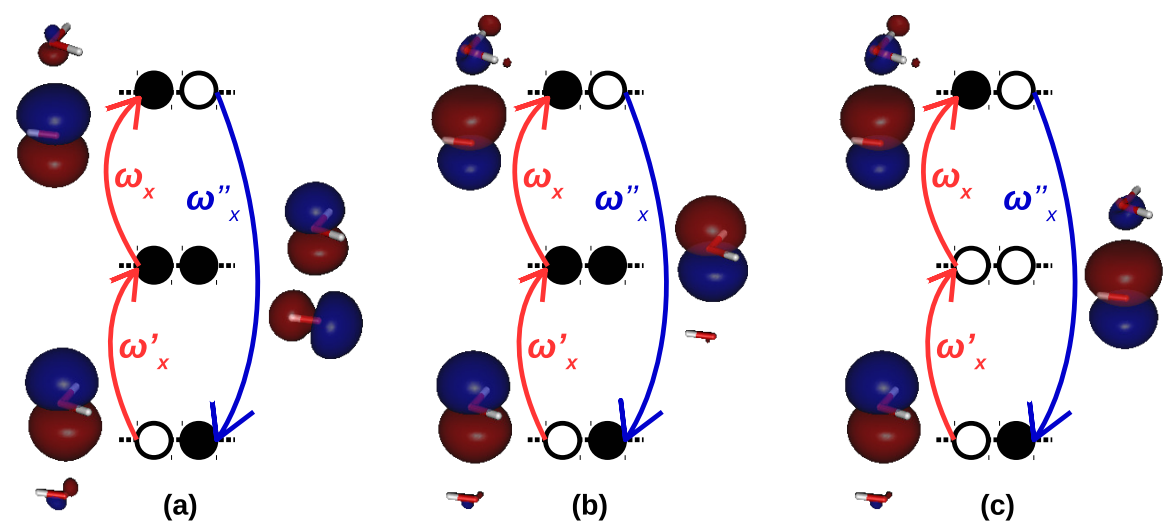

FIG. 5. The important orbital channels for para-nitroaniline in the SFG process for different sets of photon energies obtained from the NO analyses of corresponding response 1PDMs. (a) The $p_{x}\left(\mathrm{H}_{2} \mathrm{O}\right) \stackrel{\omega_{x}^{\prime}}{\longrightarrow} \sigma(\mathrm{OH} \cdot)+p_{x}\left(\mathrm{H}_{2} \mathrm{O}\right) \stackrel{\omega_{x}}{\longrightarrow} p_{x}(\mathrm{OH} \cdot) \stackrel{-\omega_{x}^{\prime \prime}}{\longrightarrow} p_{x}\left(\mathrm{H}_{2} \mathrm{O}\right)$ orbital channel. (b) The $p_{x}\left(\mathrm{H}_{2} \mathrm{O}\right) \stackrel{\omega_{x}^{\prime}}{\longrightarrow} p_{x}\left(\mathrm{H}_{2} \mathrm{O}\right) \stackrel{\omega_{x}}{\longrightarrow} p_{x}\left(\mathrm{OH}^{\cdot}\right) \stackrel{-\omega_{x}^{\prime \prime}}{\longrightarrow} p_{x}\left(\mathrm{H}_{2} \mathrm{O}\right)$ orbital channel. (c) The $p_{x}\left(\mathrm{H}_{2} \mathrm{O}\right) \stackrel{\omega_{x}^{\prime}}{\longrightarrow} p_{x}(\mathrm{OH} \cdot) \stackrel{\omega_{x}}{\longrightarrow} p_{x}(\mathrm{OH} \cdot) \stackrel{-\omega_{x}^{\prime \prime}}{\longrightarrow} p_{x}\left(\mathrm{H}_{2} \mathrm{O}\right)$ orbital channel. $\omega_{x}$ and $\omega_{x}^{\prime}$ are the absorbed photon energies and $\omega_{x}^{\prime \prime}$ is the energy of the sum-frequency photon.

larger for the latter SFG process indicating that its two-photon virtual state has larger charge-transfer character compared to that of the former SFG process.

\section{v. CONCLUSIONS}

We presented a novel black-box approach for deriving the molecular orbital picture of the underlying SFG process of $\beta$ by characterizing the virtual one- and two-photon states in terms of response 1PDMs and NOs. This is the first study extending the concept of NOs to a third-order or quadratic response property. The approach relies on computing first- and second-order response wave functions within the framework of damped response theory. Two sets of response 1PDMs, representing the transitions to the one- and two-photon virtual states from the initial zero-order state, are computed and characterized in terms of their NOs. These NOs help characterize the one- and two-photon virtual states of $\beta$, which facilitate the visualization of the underlying SFG process. The response 1PDMs are also useful for computing quantitative wave-function metrics associated with $\beta$ such the underlying extent of delocalization, electron-hole separation, and degree of charge transfer between different parts of the system. This analytic approach is superior to traditional few-state models employed for a qualitative orbital picture of $\beta$ in which the choice of intermediate states is somewhat arbitrary leading to potential loss in accuracy. We demonstrated the utility of this tool by calculating the underlying orbital picture of $\beta$ associated with the static case and a variety of non-resonant, one-photon resonant, two-photon resonant, and doubly resonant SFG processes for cis-difluoroethene, para-nitroaniline, and hemibonded $\mathrm{OH}+\mathrm{H}_{2} \mathrm{O}$ complex. We demonstrated how the real components of the damped response 1PDMs compile the off-resonance and doubly resonant orbital channels and the imaginary components of the damped response 1PDMs compile the singly resonant orbital channels. We also demonstrated how the off-resonance channels can be dominant, il- lustrating the merits of this rigorous analysis over few-state models that are based on near-resonance consideration. Electronic structure calculations for modeling SFG spectra augmented with our wave-function analysis tool will not only help characterize the spectral features but also elucidate the impact of molecular structure and intermolecular interactions on the SFG spectra.

\section{SUPPLEMENTARY MATERIAL}

This document contains Cartesian coordinates, tabulated values of the components of the $\beta$ tensors, and tabulated wave-function analyses of response 1PDMs in terms of NOs, $d_{\mathrm{exc}}^{ \pm}, d_{h \rightarrow e}^{ \pm}$, and $\mathcal{Q}^{ \pm}$for cis-difluoroethene, para-nitroaniline, and hemibonded $\mathrm{OH}+\mathrm{H}_{2} \mathrm{O}$ complex.

\section{ACKNOWLEDGMENTS}

This work was supported by the U.S. National Science Foundation (No. CHE-1856342).

\section{CONFLICTS OF INTEREST}

A.I.K. is the president and a part-owner of Q-Chem, Inc.

\section{DATA AVAILABILITY STATEMENT}

The data that supports the findings of this study are available within the article [and its supplementary material].

\footnotetext{
${ }^{1} \mathrm{~S}$. Yamaguchi and T. Tahara, Two-photon absorption spectrum of all-trans retinal, Chem. Phys. Lett. 376, 237 (2003).

${ }^{2}$ B. Dick and G. Hohlneicher, Two-photon excitation spectroscopy of phenanthrene singlet states below $50000 \mathrm{~cm}^{-1}$, Chem. Phys. Lett. 97, 324 (1983).
} 
${ }^{3}$ A. M. Rizzuto, S. Irgen-Gioro, A. Eftekhari-Bafrooei, and R. J. Saykally, Broadband deep UV spectra of interfacial aqueous iodide, J. Phys. Chem. Lett. 7, 3882 (2016)

${ }^{4}$ H. Mizuno, A. M. Rizzuto, and R. J. Saykally, Charge-transfer-to-solvent spectrum of thiocyanate at the air/water interface measured by broadband deep ultraviolet electronic sum frequency generation spectroscopy, J. Phys. Chem. Lett. 9, 4753 (2018).

${ }^{5}$ O. Fuchs, M. Zharnikov, L. Weinhardt, M. Blum, M. Weigand, Y. Zubavichus, M. Bär, F. Maier, J. D. Denlinger, C. Heske, M. Grunze, and $\mathrm{E}$. Umbach, Isotope and temperature effects in liquid water probed by $\mathrm{x}$-ray absorption and resonant $\mathrm{x}$-ray emission spectroscopy, Phys. Rev. Lett. 100, 027801 (2008).

${ }^{6}$ L. Kjellsson, K. D. Nanda, J.-E. Rubensson, G. Doumy, S. H. Southworth, P. J. Ho, A. M. March, A. Al Haddad, Y. Kumagai, M.-F. Tu, T. Debnath, M. S. Bin Mohd Yusof, C. Arnold, W. F. Schlotter, S. Moeller, G. Coslovich, J. D. Koralek, M. P. Minitti, M. L. Vidal, M. Simon, R. Santra, Z.-H. Loh, S. Coriani, A. I. Krylov, and L. Young, Resonant inelastic $\mathrm{x}$-ray scattering reveals hidden local transitions of the aqueous $\mathrm{OH}$ radical, Phys. Rev. Lett. 124, 236001 (2020).

${ }^{7}$ E.C. Carroll, S. Berlin, J. Levitz, M.A. Kienzler, Z. Yuan, D. Madsen, D.S. Larsen, and E.Y. Isacoff, Two-photon brightness of azobenzene photoswitches designed for glutamate receptor optogenetics, Proc. Nat. Acad. Sci. 112, E776 (2015).

${ }^{8}$ A.V. Kachynski, A. Pliss, A.N. Kuzmin, T.Y. Ohulchanskyy, A. Baev, J. Qu, and P.N. Prasad, Photodynamic therapy by in situ nonlinear photon conversion, Nat. Photonics 8, 455 (2014).

${ }^{9}$ H. Peng, L. Niu, Y. Chen, L. Wu, C. Tung, and Q. Yang, Biological applications of supramolecular assemblies designed for excitation energy transfer, Chem. Rev. 115, 7502 (2015).

${ }^{10}$ W.A. Velema, W. Szymanski, and B.L. Feringa, Photopharmacology: Beyond proof of principle, J. Am. Chem. Soc. 136, 2178 (2014).

${ }^{11}$ T.Z. Teisseyre, A.C. Millard, P. Yan, J.P. Wuskell, M. Wei, A. Lewis, and L.M. Loew, Nonlinear optical potentiometric dyes optimized for imaging with 1064-nm light, J. Biomed. Opt. 12, 044001 (2007).

${ }^{12}$ S. Hunter, F. Kiamilev, S. Esener, D.A. Parthenopoulos, and P.M. Rentzepis, Potentials of two-photon based 3-D optical memories for high performance computing, Appl. Opt. 29, 2058 (1990).

${ }^{13}$ S. Kawata and Y. Kawata, Three-dimensional optical data storage using photochromic materials, Chem. Rev. 100, 1777 (2000).

${ }^{14}$ C.C. Corredor, Z.L. Huang, K.D. Belfield, A.R. Morales, and M.V. Bondar, Photochromic polymer composites for two-photon 3D optical data storage, Chem. Mat. 19, 5165 (2007).

${ }^{15} \mathrm{~J}$. Olsen and P. Jørgensen, Linear and non-linear response functions for an exact state and for MCSCF state, J. Chem. Phys. 82, 3235 (1985).

${ }^{16}$ T. Helgaker, S. Coriani, P. Jørgensen, K. Kristensen, J. Olsen, and K. Ruud, Recent advances in wave function-based methods of molecular-property calculations, Chem. Rev. 112, 543 (2012).

${ }^{17}$ R.W. Boyd, Nonlinear Optics. Elsevier, 2008.

${ }^{18}$ M. Bass, P.A. Franken, J.F. Ward, and G. Weinreich, Optical rectification, Phys. Rev. Lett. 9, 446 (1962).

${ }^{19}$ P.A. Franken, A.E. Hill, C.W. Peters, and G. Weinreich, Generation of optical harmonics, Phys. Rev. Lett. 7, 118 (1961).

${ }^{20} \mathrm{M}$. Nakano and B. Champagne, Diradical character dependences of the first and second hyperpolarizabilities of asymmetric open-shell singlet systems, J. Chem. Phys. 138, 244306 (2013).

${ }^{21}$ P.C. Ray, The effects of $\pi$-conjugation on first hyperpolarizabilities of charged NLO chromophores, Chem. Phys. Lett. 394, 354 (2004).

${ }^{22}$ R. Andreu, M.J. Blesa, L. Carrasquer, J. Garín, J. Orduna, B. Villacampa, R. Alcalá, J. Casado, M.C.R. Delgado, J.T.L. Navarrete, and M. Allain, Tuning first molecular hyperpolarizabilities through the use of proaromatic spacers, J. Chem. Phys. 127, 8835 (2005).

${ }^{23}$ T. Woller, P. Geerlings, F. De Proft, B. Champagne, and M. Alonso, Aromaticity as a guiding concept for spectroscopic features and nonlinear optical properties of porphyrinoids, Molecules 23, 1333 (2018).

${ }^{24}$ J.N. Woodford, M.A. Pauley, and C.H. Wang, Solvent dependence of the first molecular hyperpolarizability of p-nitroaniline revisited, J. Phys. Chem. A 101, 1989 (1997).

${ }^{25}$ M.M. Alam, V. Kundi, and P.P. Thankachan, Solvent effects on static polarizability, static first hyperpolarizability and one- and two-photon absorption prop erties of functionalized triply twisted möbius annulenes: A DFT study,
Phys. Chem. Chem. Phys. 18, 21833 (2016).

${ }^{26}$ K. D. Nanda and A. I. Krylov, Visualizing the contributions of virtual states to two-photon absorption cross-sections by natural transition orbitals of response transition density matrices, J. Phys. Chem. Lett. 8, 3256 (2017).

${ }^{27}$ K. D. Nanda and A. I. Krylov, A simple molecular orbital picture of RIXS distilled from many-body damped response theory, J. Chem. Phys. 152, 244118 (2020).

${ }^{28}$ A. I. Krylov, From orbitals to observables and back, J. Chem. Phys. 153, 080901 (2020).

${ }^{29}$ S. Mewes, F. Plasser, A. I. Krylov, and A. Dreuw, Benchmarking excitedstate calculations using exciton properties, J. Chem. Theory Comput. 14, 710 (2018).

${ }^{30}$ F. Plasser, M. Wormit, and A. Dreuw, New tools for the systematic analysis and visualization of electronic excitations. I. Formalism, J. Chem. Phys. 141, 024106 (2014).

${ }^{31}$ A. V. Luzanov, A. A. Sukhorukov, and V. E. Umanskii, Application of transition density matrix for analysis of excited states, Theor. Exp. Chem. 10, 354 (1976), Russian original: Teor. Eksp. Khim., 10, 456 (1974).

${ }^{32}$ A. V. Luzanov and V. F. Pedash, Interpretation of excited states using charge-transfer number, Theor. Exp. Chem. 15, 338 (1979).

${ }^{33}$ M. Head-Gordon, A. M. Grana, D. Maurice, and C. A. White, Analysis of electronic transitions as the difference of electron attachment and detachment densities, J. Phys. Chem. 99, 14261 (1995).

${ }^{34}$ F. Plasser, S. A. Bäppler, M. Wormit, and A. Dreuw, New tools for the systematic analysis and visualization of electronic excitations. II. Applications, J. Chem. Phys. 141, 024107 (2014).

${ }^{35}$ S. A. Bäppler, F. Plasser, M. Wormit, and A. Dreuw, Exciton analysis of many-body wave functions: Bridging the gap between the quasiparticle and molecular orbital pictures, Phys. Rev. A 90, 052521 (2014).

${ }^{36} \mathrm{P}$. Kimber and F. Plasser, Toward an understanding of electronic excitation energies beyond the molecular orbital picture, Phys. Chem. Chem. Phys. 22, 6058 (2020).

${ }^{37}$ M. de Wergifosse, C. G. Elles, and A. I. Krylov, Two-photon absorption spectroscopy of stilbene and phenanthrene: Excited-state analysis and comparison with ethylene and toluene, J. Chem. Phys. 146, 174102 (2017).

${ }^{38}$ K. D. Nanda and A. I. Krylov, The effect of polarizable environment on two-photon absorption cross sections characterized by the equation-ofmotion coupled-cluster singles and doubles method combined with the effective fragment potential approach, J. Chem. Phys. 149, 164109 (2018).

${ }^{39}$ B.J. Orr and J.F. Ward, Perturbation theory of the non-linear optical polarization of an isolated system, Mol. Phys. 20, 513 (1971).

${ }^{40}$ P. Norman, D. M. Bishop, H. J. A. Jensen, and J. Oddershede, Nonlinear response theory with relaxation: The first-order hyperpolarizability, J. Chem. Phys. 123, 194103 (2005).

${ }^{41}$ D. C. Comeau and R. J. Bartlett, The equation-of-motion coupled-cluster method. Applications to open- and closed-shell reference states, Chem. Phys. Lett. 207, 414 (1993).

${ }^{42}$ J. F. Stanton and R. J. Bartlett, The equation of motion coupled-cluster method. A systematic biorthogonal approach to molecular excitation energies, transition probabilities, and excited state properties, J. Chem. Phys. 98, 7029 (1993).

${ }^{43}$ Shao, Y.; Gan, Z.; Epifanovsky, E.; Gilbert, A.T.B.; Wormit, M.; Kussmann, J.; Lange, A.W.; Behn, A.; Deng, J.; Feng, X., et al., Advances in molecular quantum chemistry contained in the Q-Chem 4 program package, Mol. Phys. 113, 184 (2015).

${ }^{44}$ A. I. Krylov and P. M. W. Gill, Q-Chem: An engine for innovation, WIREs: Comput. Mol. Sci. 3, 317 (2013).

${ }^{45}$ K. Kristensen, J. Kauczor, A. J. Thorvaldsen, P. Jørgensen, T. Kjaergaard, and A. Rizzo, Damped response theory description of two-photon absorption, J. Chem. Phys. 134, 214104 (2011).

${ }^{46}$ J. Kauczor, P. Norman, O. Christiansen, and S. Coriani, Communication: A reduced-space algorithm for the solution of the complex linear response equations used in coupled cluster damped response theory, J. Chem. Phys. 139, 211102 (2013).

${ }^{47}$ K. D. Nanda, M. L. Vidal, R. Faber, S. Coriani, and A. I. Krylov, How to stay out of trouble in RIXS calculations within the equation-of-motion coupled-cluster damped response theory framework? Safe hitchhiking in the excitation manifold by means of core-valence separation, Phys. Chem. Chem. Phys. 22, 2629 (2020). 
${ }^{48}$ K. D. Nanda and A. I. Krylov, Two-photon absorption cross sections within equation-of-motion coupled-cluster formalism using resolution-ofthe-identity and Cholesky decomposition representations: Theory, implementation, and benchmarks, J. Chem. Phys. 142, 064118 (2015).

${ }^{49}$ K. D. Nanda and A. I. Krylov, Static polarizabilities for excited states within the spin-conserving and spin-flipping equation-of-motion coupled-cluster singles and doubles formalism: Theory, implementation, and benchmarks, J. Chem. Phys. 145, 204116 (2016).

${ }^{50} \mathrm{H}$. Sekino and R. J. Bartlett, A linear response, coupled-cluster theory for excitation energy, Int. J. Quant. Chem. 26, 255 (1984).

${ }^{51}$ H. Koch and P. Jørgensen, Coupled cluster response functions, J. Chem. Phys. 93, 3333 (1990).

${ }^{52}$ H. Koch, H.J.Aa. Jensen, P. Jørgensen, and T. Helgaker, Excitation energies from the coupled clusters singles and doubles linear response functions (CCSDLR). Applications to Be, $\mathrm{CH}^{+}, \mathrm{CO}$, and $\mathrm{H}_{2} \mathrm{O}$, J. Chem. Phys. 93, 3345 (1990).

${ }^{53}$ P. B. Rozyczko, S. A. Perera, M. Nooijen, and R. J. Bartlett, Correlated calculations of molecular dynamic polarizabilities, J. Chem. Phys. 107,
6736 (1997).

${ }^{54}$ P. Rozyczko and R.J. Bartlett, Frequency dependent equation-of-motion coupled cluster hyperpolarizabilities: Resolution of the discrepancy between theory and experiment for HF?, J. Chem. Phys. 107, 10823 (1997).

${ }^{55}$ C. Hättig, H. Koch, and P. Jørgensen, Comment on "frequency-dependent equation-of-motion coupled cluster hyperpolarizabilities: Resolution of the discrepancy between theory and experiment for HF?" [J. Chem. Phys. 107, 10823 (1997)], J. Chem. Phys. 109, 3293 (1998).

${ }^{56}$ K. D. Nanda, A. I. Krylov, and J. Gauss, COMMUNICATION: The pole structure of the dynamical polarizability tensor in equation-of-motion coupled-cluster theory, J. Chem. Phys. 149, 141101 (2018).

${ }^{57}$ K. D. Nanda and A. I. Krylov, Cherry-picking resolvents: A general strategy for convergent coupled-cluster damped response calculations of corelevel spectra, J. Chem. Phys. 153, 141104 (2020).

${ }^{58}$ S. Coriani, F. Pawłowski, J. Olsen, and P. Jørgensen, Molecular response properties in equation of motion coupled cluster theory: A time-dependent perspective, J. Chem. Phys. 144, 02410 (2016).

${ }^{59}$ F. Plasser, Theodore: a toolbox for a detailed and automated analysis of electronic excited state computations, J. Chem. Phys. 152, 084108 (2020). 